\title{
Extended phase space of AdS Black Holes in Einstein-Gauss-Bonnet gravity with a quadratic nonlinear electrodynamics
}

\author{
S. H. Hendi ${ }^{1,2 *}$, S. Panahiyan ${ }^{1 \dagger}$ and M. Momennia ${ }^{1 \dagger}$ \\ 1 Physics Department and Biruni Observatory, College of Sciences, Shiraz University, Shiraz 71454, Iran \\ ${ }^{2}$ Research Institute for Astronomy and Astrophysics of Maragha (RIAAM), P.O. Box 55134-441, Maragha, Iran
}

\begin{abstract}
In this paper, we consider quadratic Maxwell invariant as a correction to the Maxwell theory and study thermodynamic behavior of the black holes in Einstein and Gauss-Bonnet gravities. We consider cosmological constant as a thermodynamic pressure to extend phase space. Next, we obtain critical values in case of variation of nonlinearity and Gauss-Bonnet parameters. Although the general thermodynamical behavior of the black hole solutions is the same as usual Van der Waals system, we show that in special case of the nonlinear electromagnetic field, there will be a turning point for the phase diagrams and usual Van der Waals is not observed. This theory of nonlinear electromagnetic field provides two critical horizon radii. We show that this unusual behavior of phase diagrams is due to existence of second critical horizon radius. It will be pointed out that the power of the gravity and nonlinearity of the matter field modify the critical values. We generalize the study by considering the effects of dimensionality on critical values and make comparisons between our models with their special sub classes. In addition, we examine the possibility of the existence of the reentrant phase transitions through two different methods.
\end{abstract}

\section{INTRODUCTION}

It is well known that black hole behaves as a thermodynamic system and it can be interpreted with a physical temperature and an entropy [1]. Finding the connection between the laws of black hole mechanics and the laws of ordinary thermodynamics is one of the remarkable achievements of theoretical physics during the last forty years. Therefore, it is very natural to study various thermodynamic aspects of black holes, such as thermal stability, phase transition and black hole evaporation. Studying phase transition in black holes would be one of the fascinating topics in this regard because this phenomena plays an important role in order to explore thermodynamic properties of various systems near the critical points. The first attempt to investigate the phase transition of the black holes has been done by Hut and Davies [2].

In general, there are four different approaches for studying the phase transition of the black holes, theoretically. First, the cosmological constant, $\Lambda$, is considered to be pressure of the system, in which its related conjugate quantity will be volume. By this consideration, the critical behavior can be studied through phase diagrams. Second, studying phase transition in black holes by using the Clausius-Clapeyron-Ehrenfest's equations [3]. Considering the analogy between the thermodynamic state variables and various black hole parameters $(V \leftrightarrow Q$ and $P \leftrightarrow-\Phi)$, puts us in a position to write down the Ehrenfest's equations for the black holes [4] and study their phase transition. Third, an alternative approach to investigate the phase transition was suggested by Ruppeiner in 1979 [5] in which proposed a geometrical way to study thermodynamical phase transitions. Fourth, investigating the phase transitions of black holes through the canonical ensemble by calculating heat capacity [6].

In this paper, we are going to investigate the phase transition of black hole solutions in the asymptotically AdS spacetime by considering the first mentioned approach (the cosmological constant as a pressure of the system) in both Einstein and Gauss-Bonnet (GB) gravities. In context of AdS/CFT correspondence, it was proposed that variation of $\Lambda$ corresponds to variation of the number of the colors on boundary of the Yang-Mills theory with chemical potential interpretation [7, 8]. There are two main reasons to investigate the asymptotically AdS black holes. First reason is that the AdS/CFT correspondence attracts attentions to the physics of asymptotically AdS black holes in recent years; the main focus is on understanding strongly coupled thermal field theories living on the AdS boundary. Even from a bulk perspective such black holes and their thermodynamics which exhibits various phase transitions, are quite interesting. Second, the behavior of the black hole phase transition in the asymptotically AdS spacetime is like the Van der Waals liquid/gas 9, 10], and also, is different from those of black holes in the flat space [11, 12]. Studying thermodynamic behavior of black holes in an asymptotically AdS spacetime has been done first by Hawking and Page in 1983 [13]. After that, the critical behaviors of the black holes by including the cosmological constant as a thermodynamic pressure have been investigated in [8, 9]. In this approach, the black hole mass $M$ is considered as

\footnotetext{
* email address: hendi@shirazu.ac.ir

$\dagger$ email address: sh.panahiyan@gmail.com

$\ddagger$ email address: momennia1988@gmail.com
} 
the Enthalpy of the system. Studying phase transition of black holes with Einstein gravity has been done in many literatures [14]. In addition, the critical behavior of charged AdS-GB black holes has been investigated in [15 17].

One the other hand, in electrodynamic point of view, the self-energy of a point-like charge has a divergency at the origin. In order to remove this singularity, Born and Infeld introduced an interesting kind of nonlinear electrodynamics (NED) in 1934 [18]. Coupling of NED with the gravity was first done by Hoffmann [19]. The effects of Born-Infeld NED coupled to the gravitational field have been studied in various contexts such as superconductors [20], wormholes [21, 22], static black holes [23] and rotating black objects [24]. In addition, another motivation for considering BornInfeld NED comes from the fact that it naturally arises in the low-energy limit of the heterotic string theory [25]. Recently, two different Born-Infeld types of NED have been introduced by Soleng [26] and Hendi [27]. The Soleng Lagrangian has a logarithmic form and, like Born-Infeld theory, removes divergency of the electric field while the Lagrangian proposed by Hendi has an exponential form and does not cancel the divergency of the electric field but its singularity is much weaker than that in Maxwell theory. Investigation of black object solutions coupled to these two nonlinear fields has been done in [22, 28]. The Lagrangian of mentioned Born-Infeld type nonlinear theories, for weak nonlinearity, can be written with the following form

$$
\mathcal{L}(\mathcal{F})=-\mathcal{F}+\beta \mathcal{F}^{2}+O\left(\beta^{2}\right),
$$

where $\beta$ is proportional to the inverse value of square nonlinearity parameter in Born-Infeld-type theories, so it gets just positive values. In Eq. (11), $\mathcal{F}=F_{\mu \nu} F^{\mu \nu}$ is the Maxwell invariant, $F_{\mu \nu}=\partial_{\mu} A_{\nu}-\partial_{\nu} A_{\mu}$ is the electromagnetic field tensor and $A_{\mu}$ is the gauge potential. In addition, $\beta$ denotes nonlinearity parameter which is small. For $\beta \longrightarrow 0, \mathcal{L}(\mathcal{F})$ reduces to the standard Maxwell Lagrangian, $\mathcal{L}_{\text {Maxwell }}(\mathcal{F})=-\mathcal{F}$, as it should be. In this paper, we take into account the Eq. (11) as a NED source coupled to the Einstein and GB gravities and investigate the effects of nonlinearity on the properties of the phase transition.

It is worthwhile to mention the motivations for considering the NED Lagrangian and specially Lagrangian (11). Nonlinear field theories are of interest to different branches of mathematical physics because most physical systems are inherently nonlinear in the nature. The main reason to consider NED comes from the fact that these theories are considerably richer than the Maxwell field and in special case they reduce to the linear Maxwell theory. Various limitations of the Maxwell theory, such as description of the self-interaction of virtual electron-positron pairs [29] and the radiation propagation inside specific materials [30], motivate one to consider NED [29]. Besides, NED improves the basic concept of gravitational redshift and its dependency of any background magnetic field as compared to the well-established method introduced by standard general relativity. In addition, it was recently shown that NED objects can remove both of the big bang and black hole singularities [31 33]. Moreover, from astrophysical point of view, one finds that the effects of NED become indeed quite important in superstrongly magnetized compact objects, such as pulsars and particular neutron stars (also the so-called magnetars and strange quark magnetars) [34]. Also, since the gravitational redshift of magnetized compact objects is connected to the mass-radius relation of the objects, it is important to note that NED affects the mass-radius relation of the objects. It is worthwhile to mention that one can find regular black hole solutions of the Einstein field equations coupled to a suitable NED [31, 32]. In addition, an interesting property which is common to all the NED models is that these models satisfy the zeroth and first laws of black hole mechanics. The appropriate world-volume dynamics on a curved $D 3$-brane may provide a plausible frame-work at Planck scale by incorporating the Einstein-NED. At this point, elimination of strong intrinsic curvature in the regime by the strong nonlinearity in the $U(1)$ gauge theory is remarkable [32, 35]. From the point of view of AdS/CFT correspondence in hydrodynamic models, it has been shown that, unlike gravitational correction, higherderivative terms for abelian fields in the form of NED do not affect the ratio of shear viscosity to entropy density [36]. Motivated by the recent results mentioned above and the fact that we accepted NED as a generalization of the Maxwell theory, it is natural to apply NED theories for charged objects such as black holes.

Now, we focus on motivations of considering the nonlinear term of the electromagnetic field perturbatively. Although various theories of NED have been created with different primitive motivations, only for the weak nonlinearity (Eq. (11), they contain physical and experimental importances. As we know, using the Maxwell theory in various branches leads to near accurate or acceptable consequences. So, in transition from the Maxwell theory to NED, the logical decision is to consider the effects of weak nonlinearity variations, not strong ones. This means that, one can expect to obtain precise physical results with experimental agreements, provided one regards the nonlinearity as a correction to the Maxwell field. On the other hand, several reasonable papers have been published by considering Eq. (1) as an effective Lagrangian of electrodynamics 29, 37 42]. Heisenberg and Euler have shown that quantum corrections lead to nonlinear properties of vacuum [29]. Also, it was proved that in the low energy limit of heterotic string theory, a quartic correction of the Maxwell field strength tensor appears [37]. So it is natural to consider Eq. (1) as an effective and suitable Lagrangian of electrodynamics instead of the Maxwell one. Investigating the effects of nonlinearity parameter of Eq. (11) coupled to the Einstein, GB and third order Lovelock gravities have been done in [38, 39], 40, 41] and [42], respectively. In this paper, we are dealing with stringy corrected electrodynamics, and therefore, obtained results are applicable in context of string theory. In order to separate the valid domains of the 
stringy corrected and classical Born-Infeld theories, we will present a limiting point (vertical line in the figures).

Motivations for considering GB gravity can be found in literature. For example, we refer the reader to the interesting nontrivial causal structure of GB gravity with superluminal graviton modes [43]. Finally, we should note that although most of thermodynamic works in black hole physics related to asymptotically AdS solutions, there were some attempts to investigate thermodynamical behavior of dS black holes [44].

The outline of our paper is as follows. Section II is devoted to introduction to Einstein and GB black hole solutions and their conserved quantities. Next, we extend the phase space by considering cosmological constant as thermodynamic pressure and calculate critical values and then we plot diagrams for different cases. We give a detailed discussion regarding diagrams, their physical interpretations, and the effects of both nonlinear electromagnetic and gravitational parameters. We finish our paper with some closing remarks.

\section{FIELD EQUATIONS AND CONSERVED QUANTITIES}

In order to study phase transition of Einstein-Gauss-Bonnet gravity in presence of a generalized nonlinear electromagnetic field, one can employ the following Lagrangian

$$
\mathcal{L}_{\text {tot }}=\mathcal{L}_{E N}-2 \Lambda+\alpha \mathcal{L}_{G B}+\mathcal{L}(\mathcal{F})
$$

where the Lagrangian of Einstein gravity is the Ricci scalar, $\mathcal{L}_{E N}=\mathcal{R}$, and $\Lambda$ is the negative cosmological constant. In third term of Eq. (2), $\alpha$ is the GB coefficient with dimension (Length) ${ }^{2}$ and $\mathcal{L}_{G B}$ is the Lagrangian of GB gravity with following form

$$
\mathcal{L}_{G B}=R_{a b c d} R^{a b c d}-4 R_{a b} R^{a b}+\mathcal{R}^{2} .
$$

Using variational method, we obtain the following field equations

$$
\begin{gathered}
G_{a b}^{E}+\Lambda g_{a b}+\alpha G_{a b}^{G B}=\frac{1}{2} g_{a b} \mathcal{L}(\mathcal{F})-2 \mathcal{L}_{\mathcal{F}} F_{a c} F_{b}^{c}, \\
\partial_{a}\left(\sqrt{-g} \mathcal{L}_{\mathcal{F}} F^{a b}\right)=0,
\end{gathered}
$$

where $G_{a b}^{E}$ is the Einstein tensor, $G_{a b}^{G B}=2\left(R_{a c d e} R_{b}^{c d e}-2 R_{a c b d} R^{c d}-2 R_{a c} R_{b}^{c}+\mathcal{R} R_{a b}\right)-1 / 2 \mathcal{L}_{G B} g_{a b}$ and $L_{\mathcal{F}}=$ $d \mathcal{L}(\mathcal{F}) / d \mathcal{F}$.

Now, we are interested in studying topological black holes and their phase diagrams, therefore, we employ the following static metric

$$
d s^{2}=-f(r) d t^{2}+\frac{d r^{2}}{f(r)}+r^{2} d \Omega_{n-1}^{2}
$$

in which

$$
d \Omega_{n-1}^{2}=\left\{\begin{array}{cl}
d \theta_{1}^{2}+\sum_{i=2}^{n-1} \prod_{j=1}^{i-1} \sin ^{2} \theta_{j} d \theta_{i}^{2} & k=1 \\
d \theta_{1}^{2}+\sinh ^{2} \theta_{1} d \theta_{2}^{2}+\sinh ^{2} \theta_{1} \sum_{i=3}^{n-1} \prod_{j=2}^{i-1} \sin ^{2} \theta_{j} d \theta_{i}^{2} & k=-1 \\
\sum_{i=1}^{n-1} d \theta_{i}^{2} & k=0
\end{array}\right.
$$

with volume $\omega_{n-1}$.

We use Eq. (5) and mentioned metric to obtain radial electromagnetic field tensor as [38, 41]

$$
F_{t r}=\frac{q}{r^{n-1}}-\frac{4 q^{3} \beta}{r^{3 n-3}}+O\left(\beta^{2}\right)
$$

In order to find Einstein solutions one can use two methods: one, by putting $\alpha=0$ and using mentioned field equations. Second approach is obtaining GB metric function through use of field equations and series expanding it for small values of GB parameter. In order to give more specific details, we use the second approach. Therefore, in case of GB gravity, one can obtain metric function in form of [41]

$$
f(r)=k+\frac{r^{2}}{2 \alpha^{\prime}}(1-\sqrt{\Psi(r)}),
$$


with

$$
\Psi(r)=1+\frac{8 \alpha^{\prime}}{n(n-1)}\left(\Lambda+\frac{n(n-1) m}{2 r^{n}}-\frac{n q^{2}}{(n-2) r^{2 n-2}}+\frac{2 n q^{4} \beta}{r^{4 n-4}(3 n-4)}\right)+O\left(\beta^{2}\right),
$$

where $m$ is an integration constant that is related to mass and $\alpha^{\prime}=(n-2)(n-3) \alpha$. It is evident that for case of small values of nonlinearity, the metric function will lead to the GB-Maxwell gravity. As for Einstein gravity, series expanding of GB metric function for small values of $\alpha^{\prime}$ will lead to

$$
f(r)=f_{E N}-\frac{4 q^{4}}{(n-1)(3 n-4) r^{4 n-6}} \beta+\frac{f_{E N}^{2}}{r^{2}} \alpha^{\prime}+O\left(\alpha^{\prime} \beta, \alpha^{\prime 2}, \beta^{2}\right),
$$

where the metric function of Einstein-Maxwell gravity is

$$
f_{E N}=k-\frac{2 \Lambda r^{2}}{n(n-1)}-\frac{m}{r^{n-2}}+\frac{2 q^{2}}{(n-1)(n-2) r^{2 n-4}} .
$$

Next step is devoted to calculating conserved quantities. In general, for both Einstein and GB gravities one can find total mass of black hole in form of [38, 41]

$$
M=\frac{\omega_{n-1}(n-1) m}{16 \pi} .
$$

It is notable that, although the form of total mass in GB and Einstein gravities seems to be the same, its value is different for Einstein and GB branches $(k \neq 0)$. In other words, the geometrical mass for GB gravity will be

$$
m_{G B}=k\left(k \alpha^{\prime}+r_{+}^{2}\right) r_{+}^{n-4}-\frac{2 r_{+}^{n} \Lambda}{n(n-1)}+\frac{2 q^{2}}{(n-1)(n-2) r_{+}^{n-2}}-\frac{4 q^{4} \beta}{(n-1)(3 n-4) r_{+}^{3 n-4}}+O\left(\beta^{2}\right),
$$

where $r_{+}$satisfies $f\left(r=r_{+}\right)=0$, and Eq. (14) reduces to geometrical mass for Einstein gravity in case of $\alpha^{\prime}=0$.

Previously, it was seen that obtained metric functions are representing black holes with essential singularity located at $r=0$. Geometrical properties of the solutions were investigated in [38, 41] and it was shown that these solutions can be interpreted as asymptotically AdS black holes. Therefore, by using the definition of surface gravity and its relation with Hawking temperature we find temperature of these two black holes as [41]

$$
T=\frac{k(n-1)(n-2) r_{+}^{4 n-6}\left(1+\frac{(n-4) \alpha^{\prime}}{(n-2) r_{+}^{2}}\right)-2 r_{+}^{4 n-4} \Lambda-2 r_{+}^{2 n-2} q^{2}+4 q^{4} \beta}{4 \pi(n-1) r_{+}^{4 n-5}\left(1+\frac{2 k \alpha^{\prime}}{r_{+}^{2}}\right)}+O\left(\beta^{2}\right),
$$

where in order to find temperature of Einstein gravity, it is sufficient to set $\alpha^{\prime}=0[38]$.

Due to the fact that solutions are asymptotically AdS, in order to find entropy of these two gravities, one can use Gibbs-Duhem relation. Therefore, we obtain the following relation [41]

$$
S=\frac{V_{n-1}}{4}\left(1+\frac{2(n-1) \alpha^{\prime}}{(n-3) r_{+}^{2}} k\right) r_{+}^{n-1},
$$

where in order to find entropy related to Einstein gravity, one should set GB parameter to zero [38].

Generally, as one can see, the topological structure of spacetime modifies the amount of contribution of GB parameter, for the GB gravity. Although the presence of GB gravity in flat case is evident in metric function, regarding to conserved and thermodynamic quantities no contribution of GB gravity was seen and obtained values are same as Einstein gravity. Considering this fact and equation of Gibbs free energy, the thermodynamic behavior and phase diagrams of GB and Einstein gravities in case of flat horizon are same. In addition, it was shown that [38, 41] obtained conserved and thermodynamic quantities satisfy the first law of thermodynamics with the following form

$$
d M=T d S+\Phi d Q
$$

where $Q=\frac{q}{4 \pi}$ and $\Phi=\left.\int F_{t r} d r\right|_{r=r_{+}}$. 


\section{EXTENDED PHASE SPACE AND PHASE DIAGRAMS}

In order to investigate the phase structure of the solutions, we employ the approach in which the cosmological constant is a thermodynamic variable corresponding to thermodynamical pressure with the following relation

$$
P=-\frac{\Lambda}{8 \pi}
$$

This consideration could be justified due to the fact that in quantum context, fundamental fixed parameters could vary and they are not fixed. In the absence of cosmological constant, a sourceless solution of the Einstein's equation is Minkowski spacetime where the isometry transformations are governed by Poincare group. In the presence of cosmological constant, Minkowski is no longer a valid solution and it is replaced by the (anti-)de Sitter spacetime with (anti-)de Sitter group description. Considering that we are now employing the (anti-)de Sitter group for describing kinematics, the ordinary notions of energy and momentum, as well as the relationship between them and the causal structure of spacetime will be modified [47]. Therefore, we expect to see the effects of this distortion on spacetime of black holes and its corresponding thermodynamical values which is evident from calculated thermodynamical values. As one can see the conjugating thermodynamic variable to this assumption (cosmological constant as pressure) will be volume where in literature the derived volume for different types of black holes are same as that for the topology of the spacetime [14, 15]. In order to calculate the volume of these thermodynamical systems, we use the following relation

$$
V=\left(\frac{\partial H}{\partial P}\right)_{S, Q}
$$

In addition, it was proven that the Smarr formula should be extended to Lovelock gravity as well as nonlinear theories of electrodynamics [14, 16, 48]. Scaling argument was used to derive an extension of the first law and its related modified Smarr relation that includes variations in the cosmological constant, Lovelock coefficient, and also the nonlinearity parameter [14, 16, 48]. In our case, perturbative Lovelock gravity with NED, $M$ can be a function of entropy, pressure, charge, Lovelock parameter, nonlinearity coupling coefficient. Regarding the previous section, we find that those thermodynamic quantities satisfy the following differential form,

$$
d M=T d S+\Phi d Q+V d P+\mathcal{A} d \alpha+\mathcal{B} d \beta
$$

where

$$
\begin{aligned}
& \mathcal{A}=\left(\frac{d M}{d \alpha}\right)_{S, Q, P, \beta}=\frac{(n-1) k^{2} r_{+}^{n-4}}{16 \pi}, \\
& \mathcal{B}=\left(\frac{d M}{d \beta}\right)_{S, Q, P, \alpha}=-\frac{q^{4}}{4(3 n-4) \pi r_{+}^{3 n-4}} .
\end{aligned}
$$

Moreover, scaling argument helps us to obtain the generalized Smarr relation for our black hole solutions in the extended phase space

$$
M=\frac{n-1}{n-2} T S+\Phi d Q-\frac{2}{n-2} P V-\frac{2(n-1)}{(n-2)(n-3)} \mathcal{A} \alpha+\frac{2}{n-2} \mathcal{B} d \beta .
$$

We should note that the obtained relations is valid for perturbative Lovelock gravity with NED. Hereafter, we treat the cosmological constant as a thermodynamical variable, while Gauss-Bonnet coefficient and nonlinearity parameter of NED as two constants. With doing so the total finite mass of the black hole will play the role of Enthalpy and the corresponding Gibbs free energy will be in form of

$$
G=H-T S=M-T S .
$$

The obtained volume for our considered cases is

$$
V=\frac{\omega_{n-1} r_{+}^{n}}{n}
$$

which is consistent with topological structure of spherical symmetric spacetime. This result is consistent with what was derived previously [14, 15] and shows the fact that although considering GB gravity modifies the metric function and some conserved quantities of the black hole, it does not change the volume of the black hole. In other words, the 
volume of the black hole is solely dependant on the cosmological constant (pressure). Due to relation between volume and radius of the black hole, we use horizon radius (specific volume) in order to investigate the critical behavior of these systems [14, 15].

Next step will be calculating critical values. In order to do so, we use the method in which critical values are obtained through the use of $P-r_{+}$diagrams. Since the critical point is an inflection point on the critical isotherm $P-r_{+}$diagram, we use the following relations to obtain the proper equations for critical quantities

$$
\left(\frac{\partial P}{\partial r_{+}}\right)_{T}=\left(\frac{\partial^{2} P}{\partial r_{+}^{2}}\right)_{T}=0 .
$$

It will be constructive to give a short description regarding different phase diagrams and the information they contain before presenting tables and phase diagrams. $G-T$ diagrams are representing energy level of different states that phase transition takes place between them. The characteristic swallow tail that is seen in these diagrams shows the process that we know as phase transition. It also gives interesting information regarding temperature of critical points. For $T-r_{+}$diagrams, it contains information regarding critical temperature and horizon radius in which phase transition takes place. Also, it gives some insight about single state regions which in our case is small/large black holes. It also helps us to understand the effects of different parameters on critical temperature and horizon radius, and whether by changing value of a parameter, system needs more or less energy in order to have phase transition. If one is interested in studying conductor/superconductor transition that these nonlinear electromagnetic fields are representing, studying these diagrams will give more information regarding to conductivity and superconductivity regions. Studying $P-r_{+}$diagrams gives us information regarding the behavior of pressure as a function of horizon radius, and critical pressure and horizon radius of phase transition. Finally, using the fact that the free energy, temperature, and the pressure of the system are constant during the phase transition, one can plot the coexistence curve of two phases. One of the reasons for studying these diagrams is the similarity between phase structure of black holes and the Van der Waals thermodynamical systems. Here, we have used the geometric units and investigate thermodynamic behavior and critical point of the solutions, qualitatively.

Using Eq. (24) one can find $T_{c}$ in one of the equations and replace it in other equation which leads to the following relations for calculating critical horizon radius

$$
\left\{\begin{array}{cc}
k(n-2) r_{+}^{4}+8(4 n-5) \beta q^{4} r_{+}^{10-4 n}-2(2 n-3) q^{2} r_{+}^{8-2 n}=0, & \text { Einstein } \\
(12 n-48) \alpha^{2} k^{3}-12 \alpha^{\prime} k^{2} r_{+}^{2}+\left\{4(4 n-5) \beta q^{2} r_{+}^{10-4 n}-(2 n-3) r_{+}^{8-2 n}\right\} 2 q^{2} & G B \\
+\left[\left\{4(4 n-7) \beta q^{2} r_{+}^{8-4 n}-(2 n-5) r_{+}^{6-2 n}\right\} 12 q^{2} \alpha^{\prime}+(n-2) r_{+}^{4}\right] k=0, &
\end{array} .\right.
$$

As one can see, due to complexity of the obtained relation for critical horizon radius, it is not possible to find critical horizon radius analytically. Therefore, we employ the numerical method in order to calculate critical quantities and study the effects of variation of parameters in case of spherical horizon $(k=1)$. The numerical calculations show that for cases of flat $(k=0)$ and hyperbolic $(k=-1)$ horizons, the critical pressure and critical temperature for both Einstein and GB gravities are negative, so like GB-Maxwell black holes [16], the phase transition does not take place. Here, we present various tables in order to study the effects of different parameters on critical values. Next, by using the information of these tables, we plot $P-r_{+}, T-r_{+}$and $G-T$ diagrams for Einstein and GB gravities in the presence of nonlinear corrected Maxwell field (Figs. 1-10). It is notable that following results for critical pressure and temperature are obtained by using larger critical horizon radius. In order for higher orders of corrections to be small enough and do not acquire values higher than Maxwell term, we have plotted a vertical line which represents the limit for different cases.

It is notable to mention that in order to have a well-defined vacuum solution with $m=q=0$, the pressure $P$ has to satisfy the following constraint [16, 17]

$$
0 \leq \frac{64 \pi \alpha^{\prime} P}{n(n-1)} \leq 1
$$

which puts a large bound for the pressure as maximal pressure

$$
P \leq P_{\max }=\frac{n(n-1)}{64 \pi \alpha^{\prime}} .
$$

It means that only for sufficiently small pressures, the solution Eq. (91) possesses an asymptotic AdS region. However, in this paper, we choose suitable parameters for pressure to be smaller than maximal pressure everywhere. 

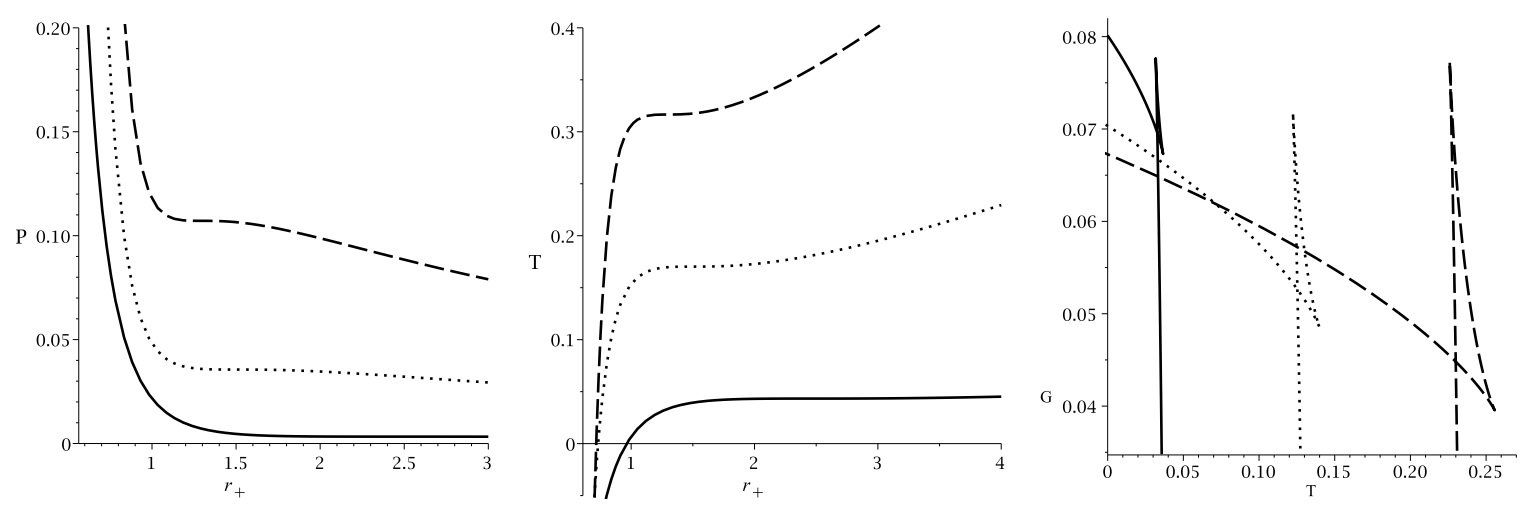

FIG. 1: Maxwell solutions for Einstein gravity: $P-r_{+}$for $T=T_{c}$ (Left), $T-r_{+}$for $P=P_{c}$ (Middle) and $G-T$ for $P=0.5 P_{c}$ (Right) diagrams for $k=1, q=1, n=3$ (continuous line), $n=4$ (dotted line) and $n=5$ (dashed line).
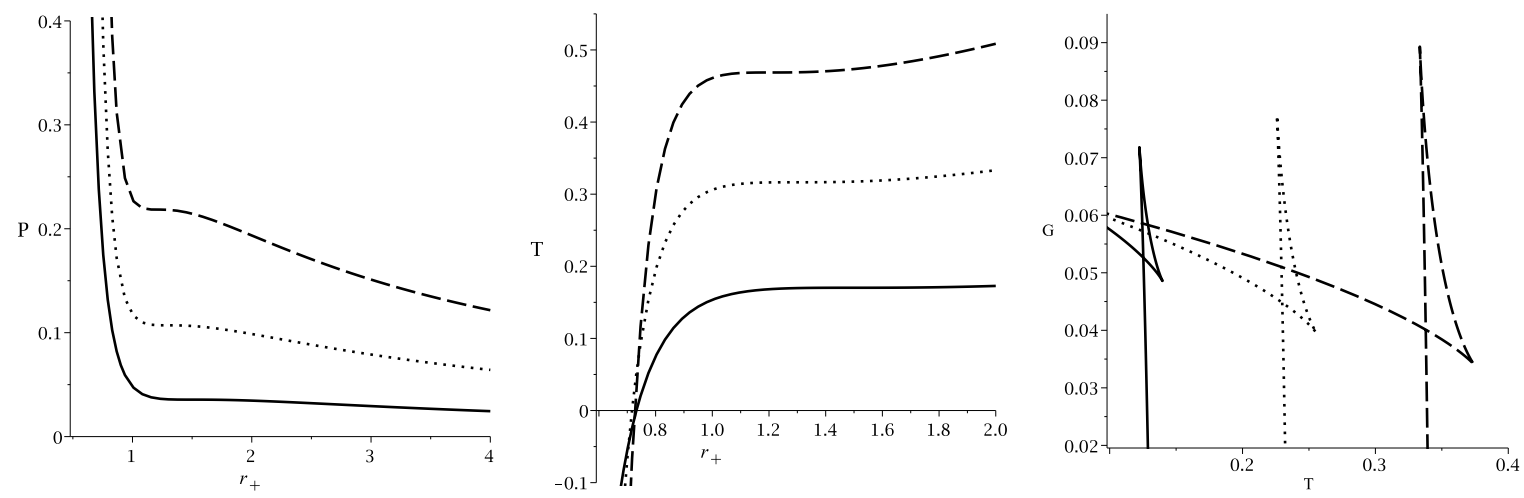

FIG. 2: Maxwell solutions for GB gravity: $P-r_{+}$for $T=T_{c}$ (Left), $T-r_{+}$for $P=P_{c}$ (Middle) and $G-T$ for $P=0.5 P_{c}$ (Right) diagrams for $k=1, q=1, \alpha^{\prime}=10^{-4}, n=4$ (continuous line), $n=5$ (dotted line) and $n=6$ (dashed line).
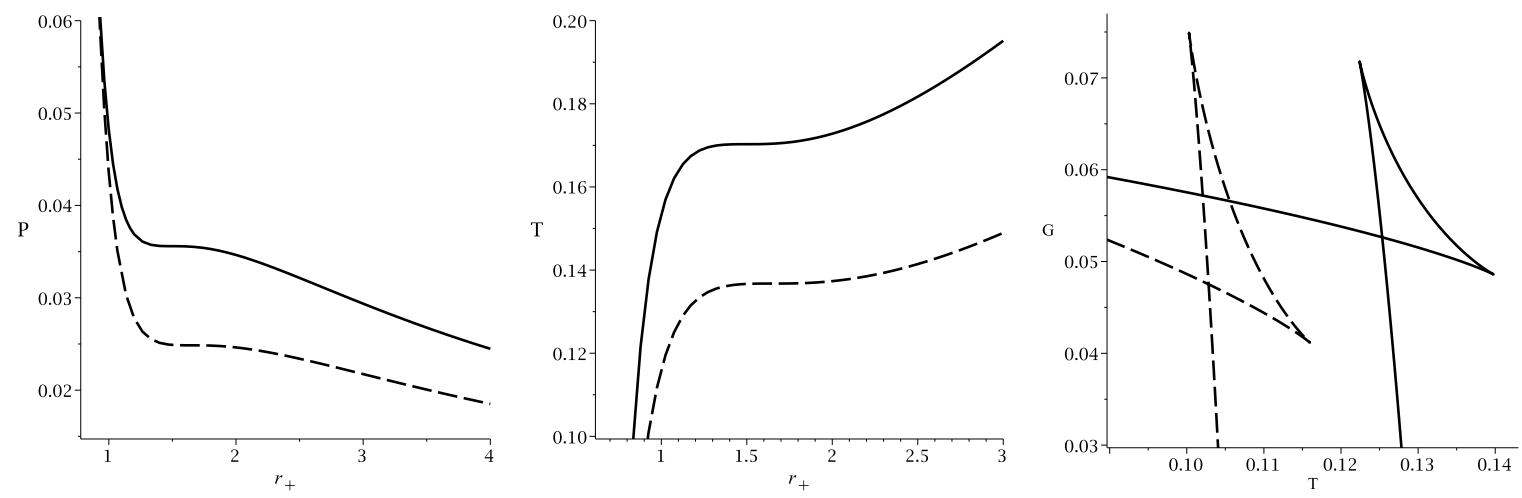

FIG. 3: Maxwell solutions for Einstein and $\boldsymbol{G B}$ gravities: $P-r_{+}$for $T=T_{c}$ (Left), $T-r_{+}$for $P=P_{c}$ (Middle) and $G-T$ for $P=0.5 P_{c}$ (Right) diagrams for $k=1, n=4, q=1, \alpha^{\prime}=0.1$ for Einstein (continuous line) and GB (dashed line) gravities. 

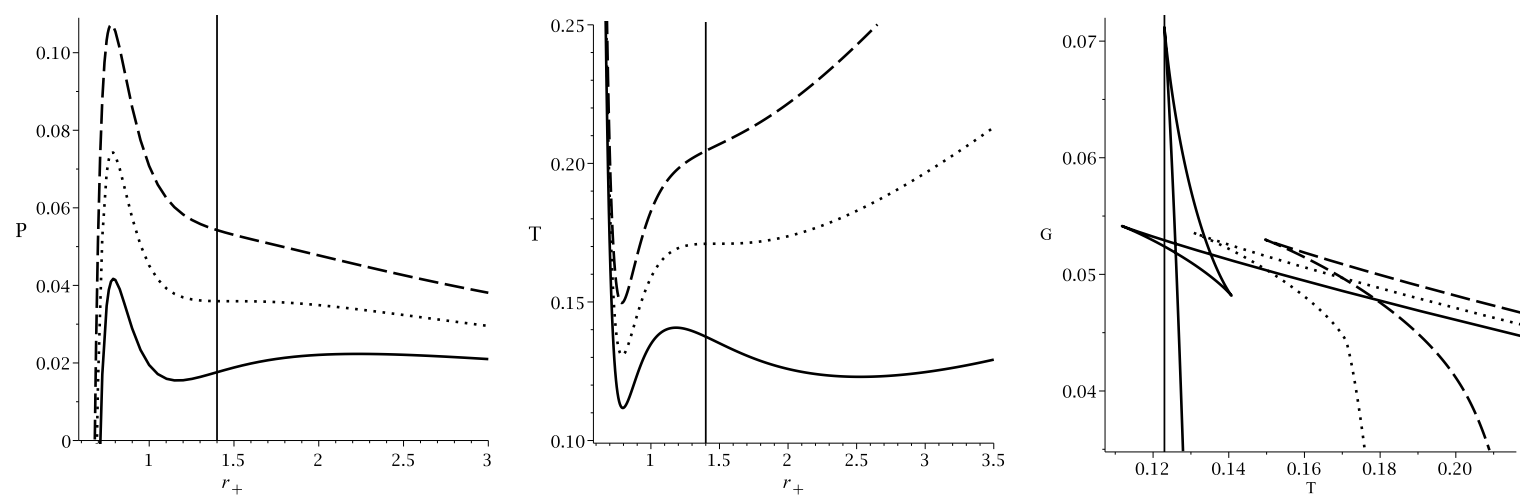

FIG. 4: Einstein solutions: $P-r_{+}$(Left), $T-r_{+}$(Middle) and $G-T$ (Right) diagrams for $k=1, n=4, q=1$ and $\beta=0.045$.

$P-r_{+}$diagram: $T=0.8 T_{c}$ (continuous line), $T=T_{c}$ (dotted line) and $T=1.2 T_{c}$ (dashed line).

$T-r_{+}$diagram: $P=0.5 P_{c}$ (continuous line), $P=P_{c}$ (dotted line) and $P=1.5 P_{c}$ (dashed line).

$G-T$ diagram: $P=0.5 P_{c}$ (continuous line), $P=P_{c}$ (dotted line) and $P=1.5 P_{c}$ (dashed line).
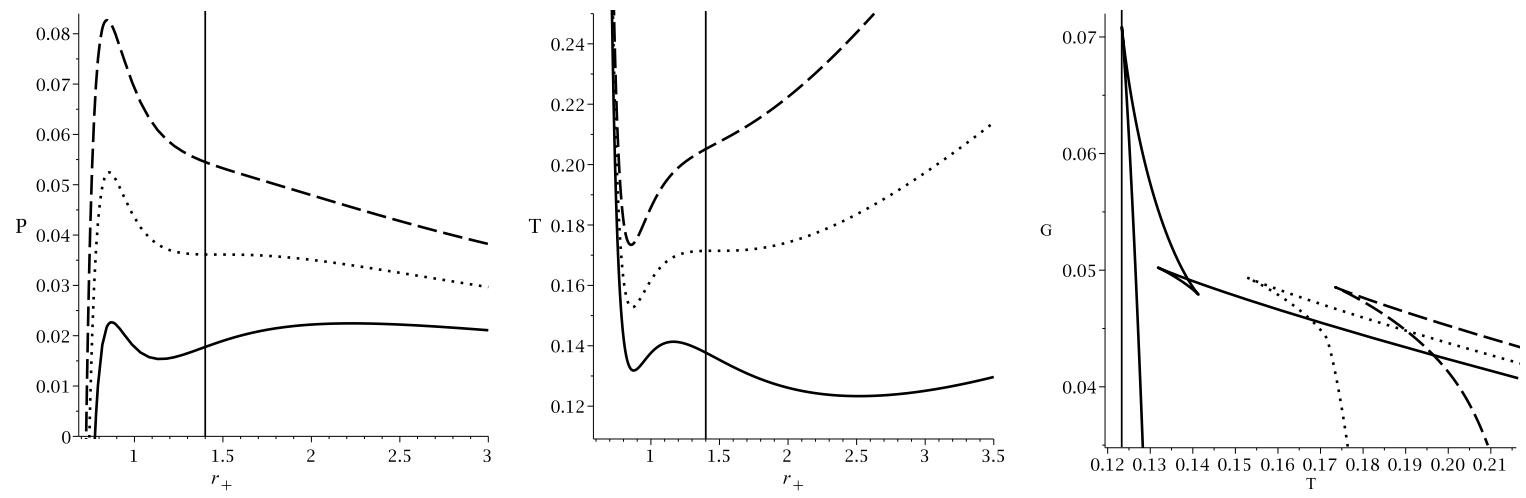

FIG. 5: Einstein solutions: $P-r_{+}$(Left), $T-r_{+}$(Middle) and $G-T$ (Right) diagrams for $k=1, n=4, q=1$ and $\beta=0.07$. $P-r_{+}$diagram: $T=0.8 T_{c}$ (continuous line), $T=T_{c}$ (dotted line) and $T=1.2 T_{c}$ (dashed line).

$T-r_{+}$diagram: $P=0.5 P_{c}$ (continuous line), $P=P_{c}$ (dotted line) and $P=1.5 P_{c}$ (dashed line).

$G-T$ diagram: $P=0.5 P_{c}$ (continuous line), $P=P_{c}$ (dotted line) and $P=1.5 P_{c}$ (dashed line).

\begin{tabular}{|c|c|c|c|c|c|c|}
\hline$\beta$ & $r_{c}$ & $\begin{array}{ll}P_{c} & \frac{P_{c} v_{c}}{T_{c}} \\
\end{array}$ & $\beta$ & $r_{c}$ & $T_{c}$ & $\frac{P_{c} v_{c}}{T_{c}}$ \\
\hline 0 & 2.44948 & 0.043300 .003310 .18750 & 0 & 1.49534 & 0.170290 .03558 & 0.31250 \\
\hline 0.00100 & $\begin{array}{l}0.31209 \\
2.44917\end{array}$ & $\begin{array}{llll}0.04331 & 0.00332 & 0.18748\end{array}$ & 0.00100 & $\begin{array}{l}0.45502 \\
1.49505\end{array}$ & 0.170300 .03559 & 0.31247 \\
\hline 0.05000 & $\begin{array}{l}0.85371 \\
2.43312 \\
\end{array}$ & $\begin{array}{llll}0.04343 & 0.00333 & 0.18569\end{array}$ & 0.05000 & $\begin{array}{l}0.89202 \\
1.47940 \\
\end{array}$ & 0.171100 .03596 & 0.31093 \\
\hline 0.10000 & $\begin{array}{l}1.03212 \\
2.41568\end{array}$ & $\begin{array}{llll}0.04356 & 0.00336 & 0.18635\end{array}$ & 0.10000 & $\begin{array}{l}1.01944 \\
1.46018\end{array}$ & 0.172010 .03639 & 0.30890 \\
\hline 0.15000 & $\begin{array}{l}1.15889 \\
2.39698\end{array}$ & $\begin{array}{llll}0.04370 & 0.00338 & 0.18569\end{array}$ & 0.15000 & $\begin{array}{l}1.11349 \\
1.43543\end{array}$ & 0.173100 .03690 & 0.30603 \\
\hline
\end{tabular}

Table 1 (left): Einstein gravity for $q=1$ and $n=3$. Table 2 (right): Einstein gravity for $q=1$ and $n=4$. 

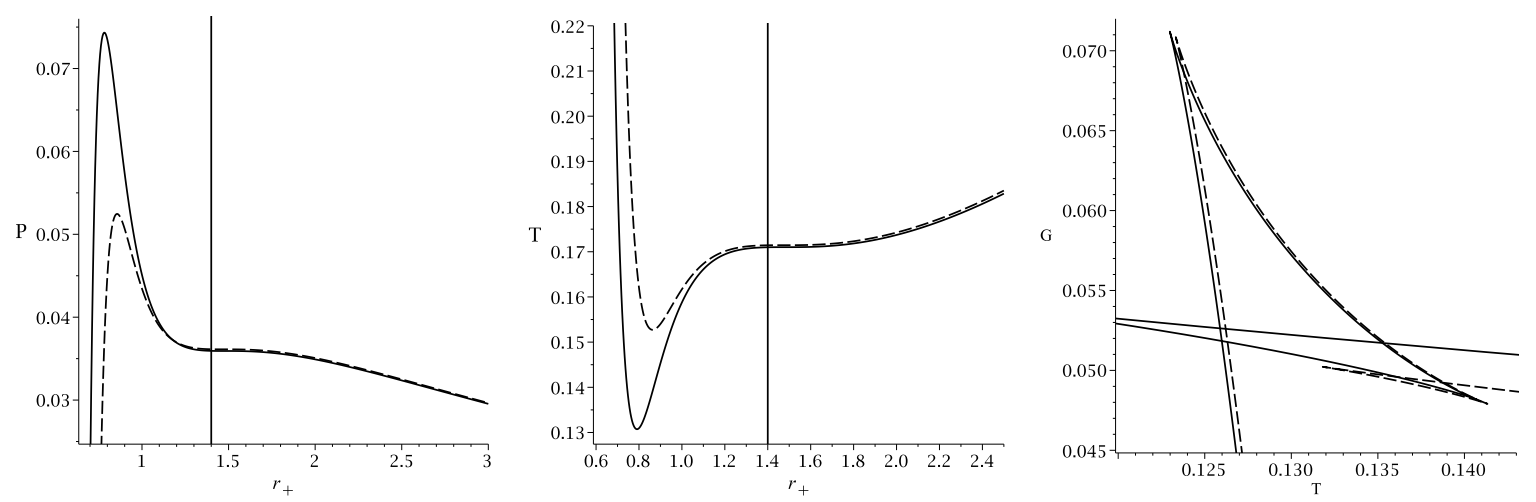

FIG. 6: Einstein solutions: $P-r_{+}$for $T=T_{c}$ (Left), $T-r_{+}$for $P=P_{c}$ (Middle) and $G-T$ for $P=0.5 P_{c}$ (Right) diagrams for $k=1, n=4, q=1, \beta=0.045$ (continuous line) and $\beta=0.07$ (dashed line).
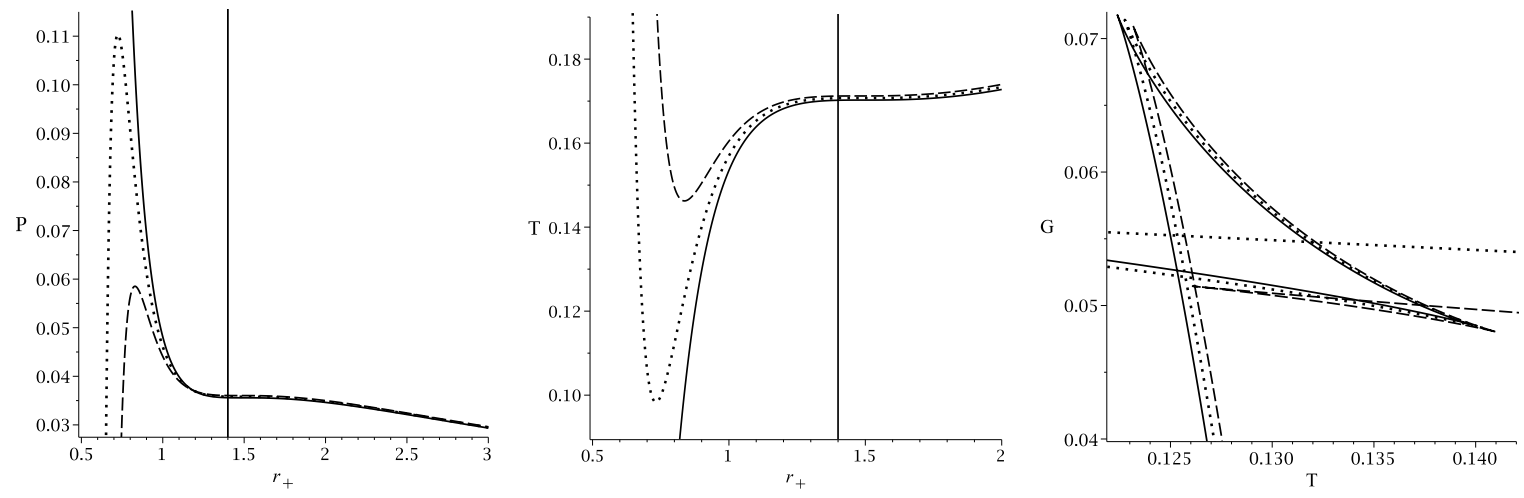

FIG. 7: $\boldsymbol{G B}$ solutions: $P-r_{+}$for $T=T_{c}$ (Left), $T-r_{+}$for $P=P_{c}$ (Middle) and $G-T$ for $P=0.5 P_{c}$ (Right) diagrams for $k=1, n=4, q=1, \alpha^{\prime}=10^{-4}$, and $\beta=0$ (continuous line), $\beta=0.03$ (dotted line) and $\beta=0.06$ (dashed line).

\begin{tabular}{|c|c|c|c|c|c|c|c|c|c|}
\hline$\beta$ & $r_{c}$ & $T_{c}$ & $P_{c}$ & $\frac{P_{c} v_{c}}{T_{c}}$ & $\beta$ & $r_{c}$ & $T_{c}$ & $P_{c}$ & $\frac{P_{c} v_{c}}{T_{c}}$ \\
\hline 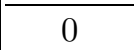 & 1.29271 & 0.31658 & 0.10714 & $\overline{0.43750}$ & 0 & 1.49550 & 0.17024 & 0.03557 & 0.31248 \\
\hline 0.00100 & $\begin{array}{l}0.55202 \\
1.29247 \\
\end{array}$ & 0.31661 & 0.10716 & 0.43746 & 0.01000 & $\begin{array}{l}0.67158 \\
1.49252 \\
\end{array}$ & 0.17040 & 0.03564 & 0.31219 \\
\hline 0.05000 & $\begin{array}{l}0.91471 \\
1.27952 \\
\end{array}$ & 0.31804 & 0.10820 & 0.43532 & 0.03000 & $\begin{array}{l}0.81326 \\
1.48626 \\
\end{array}$ & 0.17071 & 0.03579 & 0.31158 \\
\hline 0.10000 & $\begin{array}{l}1.01396 \\
1.26233\end{array}$ & 0.31978 & 0.10949 & 0.43221 & 0.05000 & $\begin{array}{l}0.89202 \\
1.47957\end{array}$ & 0.1710 & 0.03594 & 0.31091 \\
\hline 0.15000 & $\begin{array}{l}1.09170 \\
1.23597\end{array}$ & 0.32202 & 0.1111 & 0.42668 & 0.07000 & $\begin{array}{l}0.95018 \\
1.47237\end{array}$ & 0.1714 & 0.0361( & 0.31017 \\
\hline
\end{tabular}

Table 3 (left): Einstein gravity for $q=1$ and $n=5$. Table 4 (right): GB gravity for $q=1, \alpha=10^{-4}$ and $n=4$. 

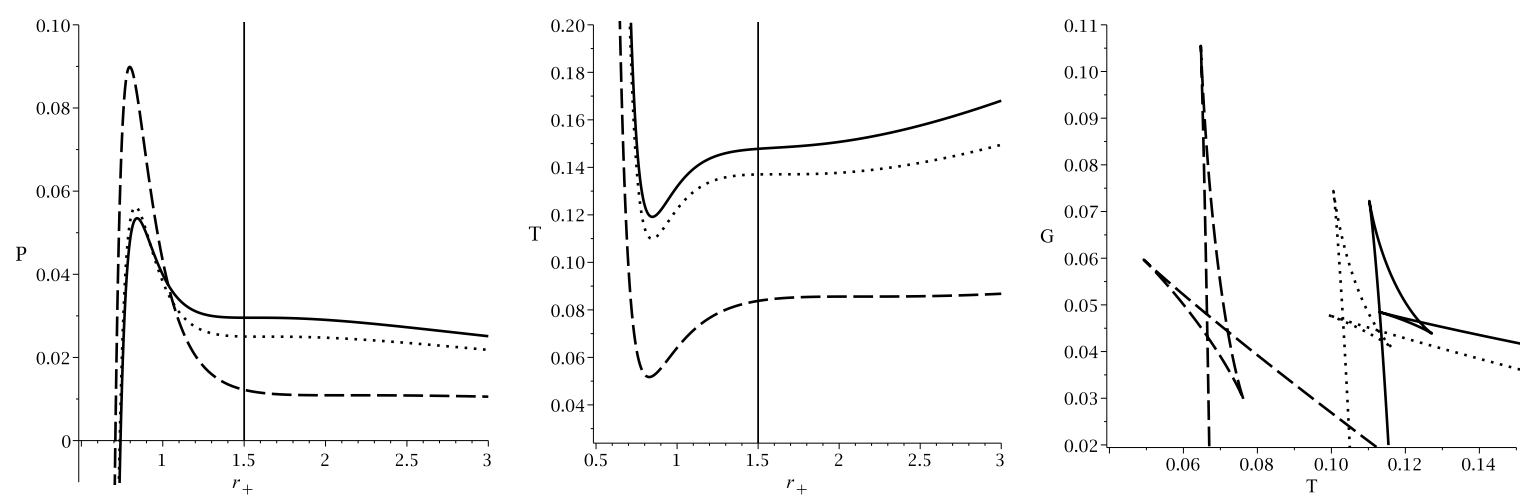

FIG. 8: $\boldsymbol{G B}$ solutions: $P-r_{+}$(Left), $T-r_{+}$(Middle) and $G-T$ (Right) diagrams for $k=1, n=4, q=1$ and $\beta=0.07$. $P-r_{+}$diagram: $T=T_{c}$ and $\alpha^{\prime}=0.05$ (continuous line), $\alpha^{\prime}=0.1$ (dotted line) and $\alpha^{\prime}=0.5$ (dashed line). $T-r_{+}$diagram: $P=P_{c}$ and $\alpha^{\prime}=0.05$ (continuous line), $\alpha^{\prime}=0.1$ (dotted line) and $\alpha^{\prime}=0.5$ (dashed line). $G-T$ diagram: $P=0.5 P_{c}$ and $\alpha^{\prime}=0.05$ (continuous line), $\alpha^{\prime}=0.1$ (dotted line) and $\alpha^{\prime}=0.5$ (dashed line).
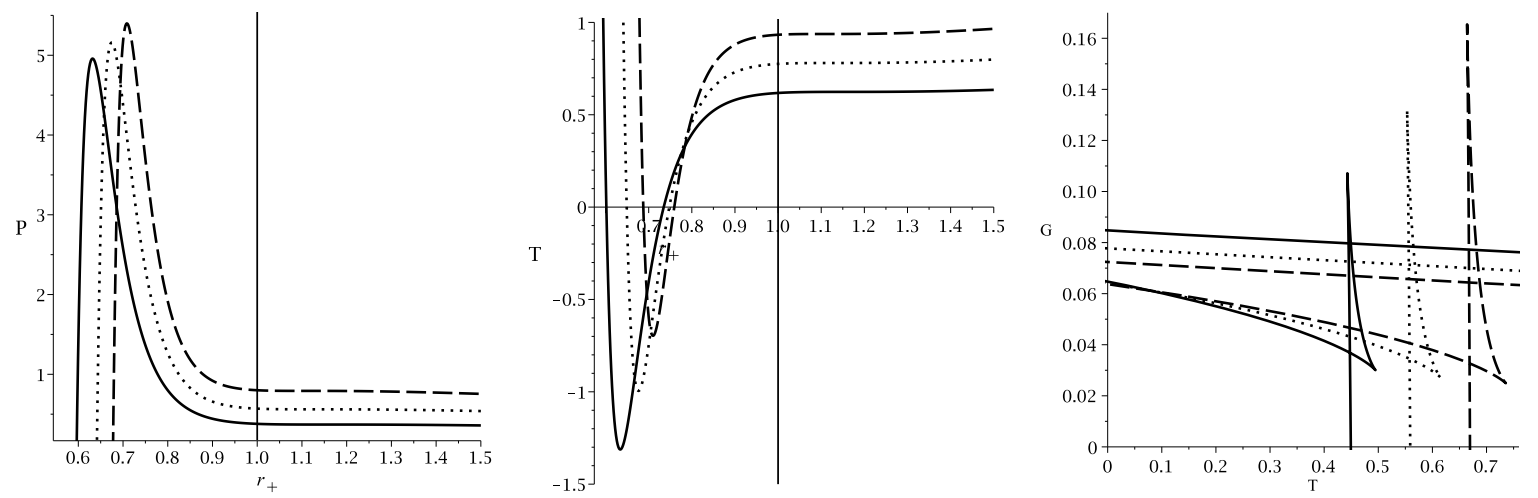

FIG. 9: Einstein solutions: $P-r_{+}$for $T=T_{c}$ (Left), $T-r_{+}$for $P=P_{c}$ (Middle) and $G-T$ for $P=0.5 P_{c}$ (Right) diagrams for $k=1, q=1, \beta=0.001, n=7$ (continuous line), $n=8$ (dotted line) and $n=9$ (dashed line).

\begin{tabular}{|ccccc|}
\hline \hline$\alpha$ & $r_{c}$ & $T_{c}$ & $P_{c}$ & $\frac{P_{c} v_{c}}{T_{c}}$ \\
\hline \hline 0.01000 & $\begin{array}{l}0.94914 \\
1.48981\end{array}$ & 0.16688 & 0.03458 & 0.30873 \\
\hline 0.05000 & $\begin{array}{l}0.94608 \\
1.55579\end{array}$ & 0.15157 & 0.02955 & 0.30333 \\
\hline 0.10000 & $\begin{array}{l}0.94374 \\
1.63116\end{array}$ & 0.13707 & 0.02499 & 0.29747 \\
\hline 0.50000 & $\begin{array}{l}0.93836 \\
2.12855\end{array}$ & 0.08560 & 0.01089 & 0.27103 \\
\hline 1.00000 & $\begin{array}{l}0.89005 \\
2.65829\end{array}$ & 0.06346 & 0.00619 & 0.25942 \\
\hline
\end{tabular}

\begin{tabular}{|ccccc|}
\hline \hline$\beta$ & $r_{c}$ & $T_{c}$ & $P_{c}$ & $\frac{P_{c} v_{c}}{T_{c}}$ \\
\hline \hline 0 & 1.49550 & 0.17024 & 0.03557 & 0.31248 \\
\hline 0.01000 & $\begin{array}{l}0.67158 \\
1.49252\end{array}$ & 0.17040 & 0.03564 & 0.31219 \\
\hline 0.03000 & $\begin{array}{l}0.81326 \\
1.48626\end{array}$ & 0.17071 & 0.03579 & 0.31158 \\
\hline 0.05000 & $\begin{array}{l}0.89202 \\
1.47957\end{array}$ & 0.17105 & 0.03594 & 0.31091 \\
\hline 0.07000 & $\begin{array}{l}0.95018 \\
1.47237\end{array}$ & 0.17140 & 0.03610 & 0.31017 \\
\hline
\end{tabular}

Table 5 (left): GB gravity for $q=1, \beta=0.07$ and $n=4$. Table 6 (right): GB gravity for $q=1, \alpha=10^{-4}$ and $n=5$. 

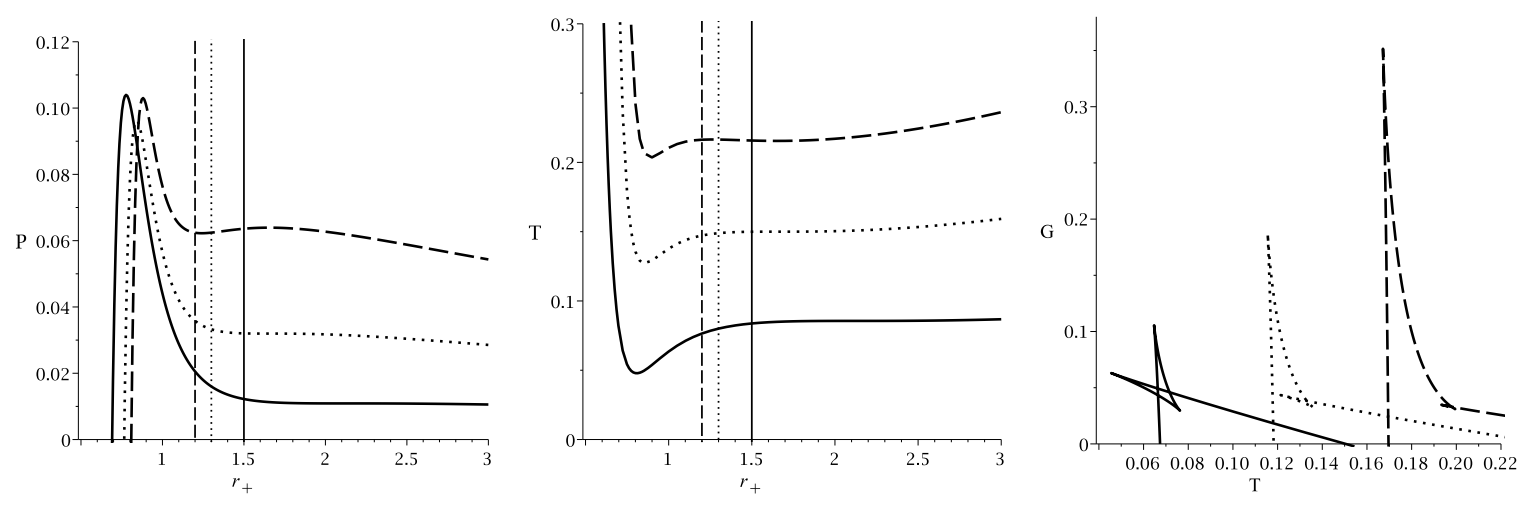

FIG. 10: $G B$ solutions: $P-r_{+}$for $T=T_{c}$ (Left), $T-r_{+}$for $P=P_{c}$ (Middle) and $G-T$ for $P=0.5 P_{c}$ (Right) diagrams for $k=1, q=1, \beta=0.06, \alpha^{\prime}=0.5, n=4$ (continuous line), $n=5$ (dotted line) and $n=6$ (dashed line).
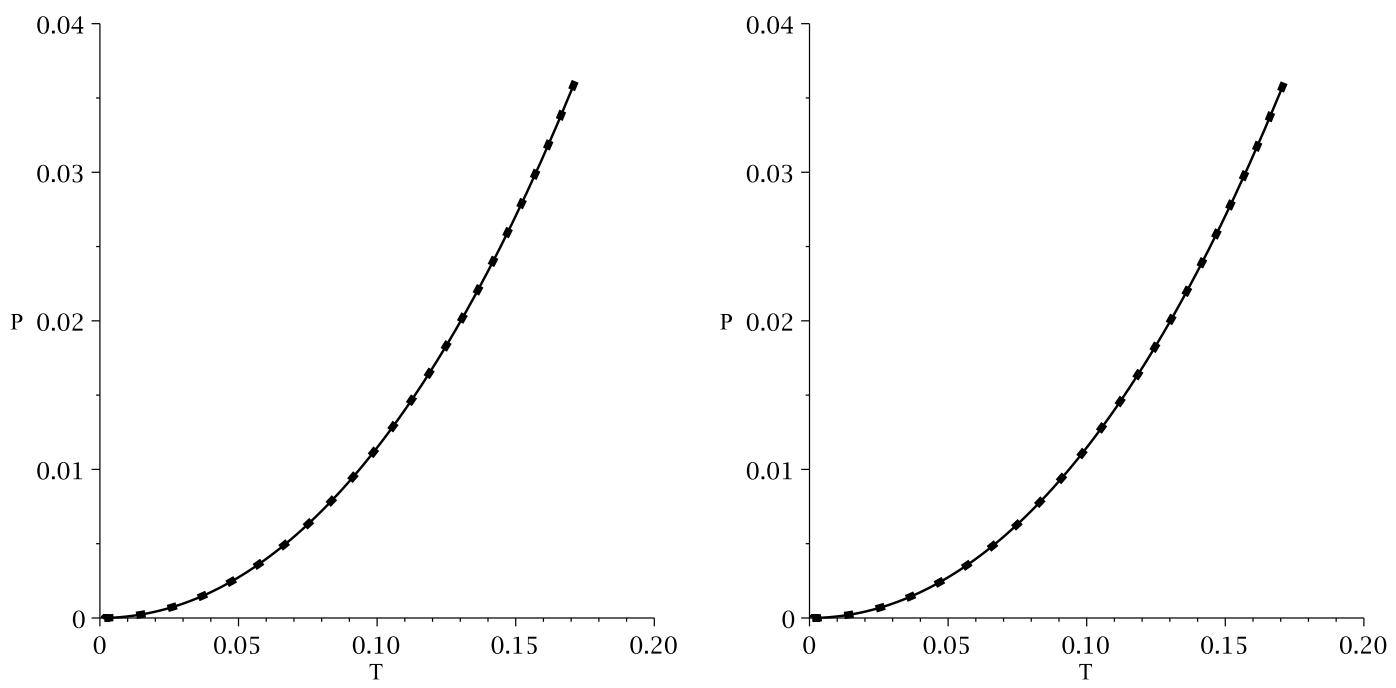

FIG. 11: Coexistence line for Einstein (Left) and GB (Right) gravities: $P-T$ for $k=1, n=4$ and $q=1$.

Left diagram: $\beta=0.045$ (continuous line) and $\beta=0.07$ (dotted line).

Right diagram: $\alpha^{\prime}=10^{-4}$ and $\beta=0$ (continuous line) and $\beta=0.06$ (dotted line).

\begin{tabular}{ccccc}
\hline \hline$\alpha$ & $r_{c}$ & $T_{c}$ & $P_{c}$ & $\frac{P_{c} v_{c}}{T_{c}}$ \\
\hline \hline 0.01000 & $\begin{array}{l}0.95884 \\
1.28530\end{array}$ & 0.30889 & 0.10367 & 0.43139 \\
\hline 0.05000 & $\begin{array}{l}0.95598 \\
1.32895\end{array}$ & 0.27681 & 0.08766 & 0.42089 \\
\hline 0.10000 & $\begin{array}{l}0.95414 \\
1.37662\end{array}$ & 0.24745 & 0.07358 & 0.40937 \\
\hline 0.50000 & $\begin{array}{l}0.95469 \\
1.65361\end{array}$ & 0.15000 & 0.03196 & 0.35241 \\
\hline 1.00000 & $\begin{array}{l}0.96227 \\
1.91323\end{array}$ & 0.11049 & 0.01837 & 0.31815 \\
\hline
\end{tabular}

Table (7): GB gravity for $q=1, \beta=0.07$ and $n=5$.

It is a well-known fact that inclusion of higher order polynomial terms (e.g. $r_{+}^{d}, d \in \mathbb{N}$ ) in the Van der Waals equation of state changes the location of critical point in thermodynamic space $(P, V, T)$, but does not change the universality class and therefore leads to the same exponents as the Van der Waals fluid (see [12, 16] for more details). 

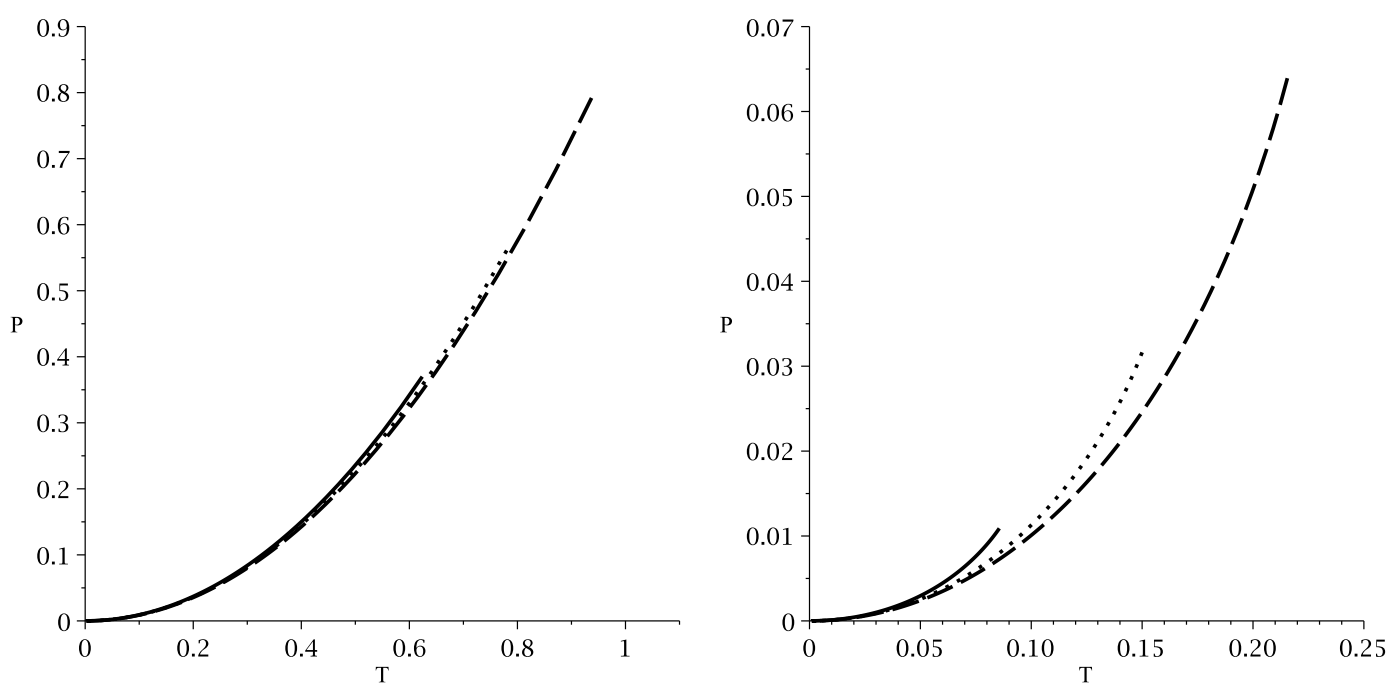

FIG. 12: Coexistence line for Einstein (Left) and GB (Right) gravities: $P-T$ for $k=1, n=4$ and $q=1$. Left diagram: $\beta=0.001$ and $n=7$ (continuous line), $n=8$ (dotted line) and $n=9$ (dashed line).

Right diagram: $\beta=0.06, \alpha^{\prime}=0.5$ and $n=4$ (continuous line), $n=5$ (dotted line) and $n=6$ (dashed line).

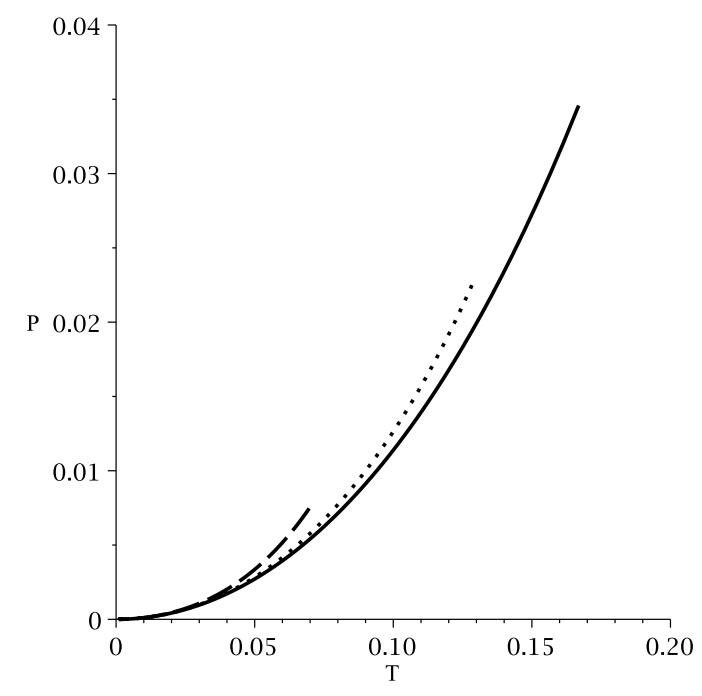

FIG. 13: Coexistence line for GB gravity: $P-T$ for $k=1, n=4, q=1, \beta=0.07$ and $\alpha^{\prime}=0.05$ (continuous line), $\alpha^{\prime}=0.1$ (dotted line) and $\alpha^{\prime}=0.5$ (dashed line).

This is different from the case of pure Lovelock gravity solutions [49], which the equation of state is no longer a polynomial form.

\section{DISCUSSION ON THE RESULTS OF DIAGRAMS}

In order to study the behavior of phase transition for these black holes in more details, we have plotted $P-r_{+}$, $T-r_{+}$and $G-T$ diagrams. For having better insight regarding the effects of correction on critical behavior of the system, we have also plotted some diagrams for the case of $\beta=0$ which is the Maxwell theory.

like Van der Waals system, the usual characteristic swallow tail is seen in black holes only in the presence of linear Maxwell field (right panels of Figs. 1 and 2). In other words, considering Maxwell electromagnetic field leads to a Van der Waals like behavior and usual phase transition. The existence and usual behavior of phase transition are also evident from studying $P-r_{+}$and $T-r_{+}$diagrams. In case of the absence of nonlinearity parameter, for $P-r_{+}$ 
(left panels of Figs. 1 and 2), pressure is a decreasing function of horizon radius but for a range of horizon radius, it is an increasing function of it which is not a physical behavior. Before and after this region there are two values of horizon with same pressure. The phase transition takes place between these two points which in case of black holes, it is small/large black hole phase transition. It is crucial to mention that, this region is only seen for case of $T \leq T_{c}$ whereas for $T>T_{c}$ pressure is only a decreasing function of horizon radius. On the other hand, for $\beta=0$ in case of $T-r_{+}$diagrams (middle panels of Figs. 1 and 2), if $P=P_{c}$ the temperature is an increasing function of horizon radius and for a region of horizon radius, temperature is fixed. This place is the region where phase transition takes place and known as subcritical isobar.

As for the effects of dimensions on the critical behavior of the system in the presence of linear Maxwell field Figs. 1 and 2 are plotted. As one can see, the swallow tail (right panels of Figs. 11 and 2), critical pressure (left panels of Figs. 1 and 2) and temperature (middle panels of Figs. 1 and 2) are increasing functions of dimension while critical horizon radius and subcritical isobars are decreasing functions of it.

In addition, for the case of absence of nonlinearity parameter, we compare critical behavior of these two gravities with each other (Fig. 3). As one can see considering GB gravity leads to increasing the size of swallow tail (right panel of Fig. 3). The place of swallow tail is also shifted to lower values of temperature. On the other hand, pressure (left panel of Fig. 3) and temperature (middle panel of Fig. 3) of the critical point are greater in Einstein gravity whereas the length of subcritical isobar and the critical horizon radius are greater in GB gravity. Also, the needed energy for the phase transition in Einstein gravity is more than GB case.

Next, we are considering $\beta \neq 0$ and plot one set of graphs for Einstein gravity for variation of nonlinearity parameter (Figs. 446) and Figs. 7 and 8 for variation of nonlinearity and GB parameters for GB gravity. Also, we plot Figs. 9 and 10 to investigate the effects of dimensions on the solutions of Einstein and GB gravities, respectively. It is notable that in these figures physical solutions exist only after the vertical line. This means that before these vertical lines the second term in Eq. (8) is not small enough (with respect to Maxwell term). The vertical lines in $P-r_{+}$ $\left(T-r_{+}\right)$and $G-T$ diagrams are interpreted as the minimum value of the authorized horizon radius and temperature, respectively. In comparing figures there are more than one vertical line. Because of overlapping of these vertical lines with the vertical line of $G-T$ diagrams, these lines are not presented.

In case of variation of nonlinearity parameter same behavior is observed for Einstein and GB gravities. For this case the following results are obtained. Interestingly, contrary to Maxwell theory, in this case $(\beta \neq 0)$, the Van der Waals like behavior is not preserved. The plotted graphs for Gibbs free energy versus temperature show the existence of a phase transition, a turning point. In other words, the characteristic swallow tail of phase transition in this nonlinear theory is modified and its shape is different from the usual thermodynamical systems. For small values of nonlinearity parameter (right panel of Fig. 4), the usual swallow tail is observed with a turning point and a minimum temperature (vertical line) which are located before swallow tail. It is evident that the minimum energy and the distance between turning point and swallow tail, are decreasing functions of $\beta$ whereas the minimum temperature is an increasing function(see Figs. 6 and 7). It is worthwhile to mention that minimum temperature and critical temperature are two different quantities.

As for the $P-r_{+}$, it is evident that the related graphs are modified like $G-T$ diagrams. First, pressure is an increasing function of horizon radius, then after a turning point, it changes into being a decreasing function of $r_{+}$. In this case, a part of the graphs shows the usual behavior of phase transition whereas there is another part which is irregular (left panels of Figs. 4 and 5). In case of $T=T_{c}$, one can find two horizon radii for critical pressure. In this case the critical horizon radius is a decreasing function of nonlinearity parameter whereas the turning point and critical pressure are increasing functions of it (Tables $1-4$ and 6 ).

Moreover, in studying $T-r_{+}$, same abnormal behavior is observed (middle panels of Figs. 4 and 5 ). Usually, we are expecting temperature to be a decreasing function of horizon radius except in place of phase transition in which temperature is fixed and horizon radius increases. This region is know as subcritical isobars. But in this case, first temperature is a decreasing function of $r_{+}$then it becomes an increasing function of it. As one can see in case of $P=P_{c}$ the subcritical isobar is observed but another value of horizon radius exists which has the same temperature as subcritical isobar. This may show that in this place phase transition takes place. The critical temperature is an increasing function of nonlinearity parameter.

Comparing the variational effects of GB and nonlinearity parameters, we find that they have opposite effects (Figs. 7 and (8). Thus, we leave out discussions of GB parameter for reasons of economy.

It is worthwhile to mention a few characteristic behavior of graphs. As one can see, in case of small values of nonlinearity parameter, the distance between the critical point and turning point is large (right panels of Figs. 4 and 5). Similarly, in case of $P-r_{+}\left(T-r_{+}\right)$the region of $r_{+}$in which pressure being increasing (decreasing) function of $r_{+}$is large too. As nonlinearity parameter increases, the distance between these points decreases in $G-T$ diagrams, and interestingly, in case of $P-r_{+}\left(T-r_{+}\right)$the region of $\mathrm{r}+$, distance between the critical horizon radius, and turning point decrease (Figs. 6] and 7). In the end, we mention that $P_{c} r_{c} / T_{c}$ is a decreasing function of nonlinearity and GB parameters. 

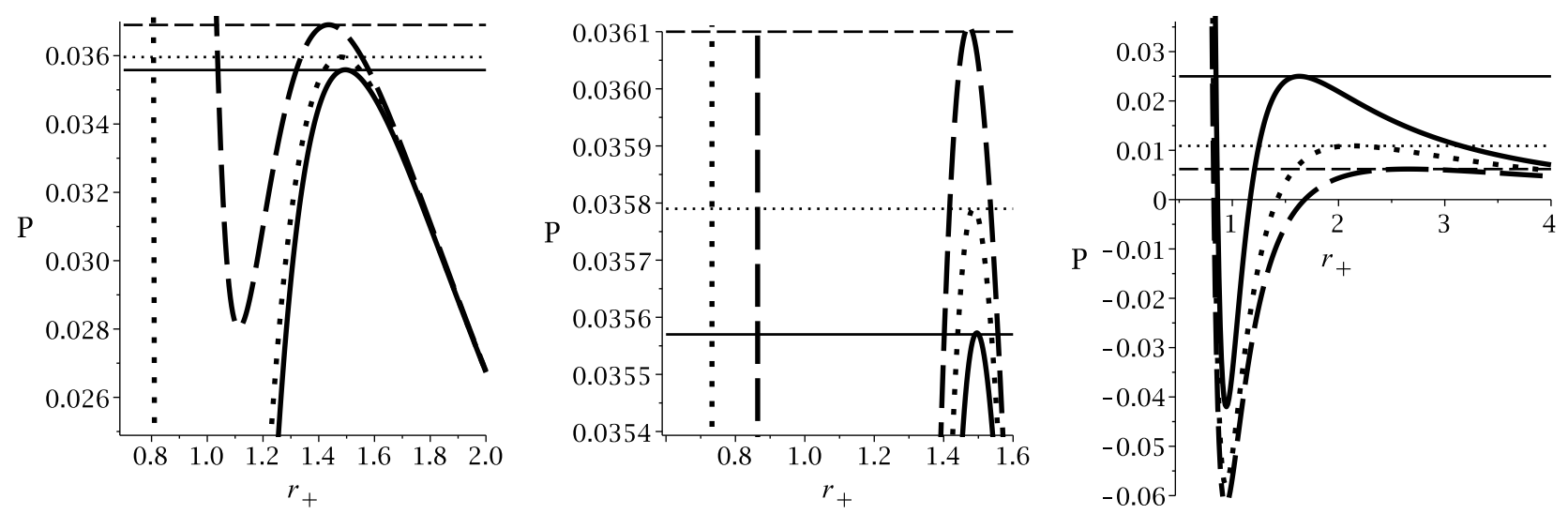

FIG. 14: $P$ versus $r_{+}$diagrams for $q=1$ and $k=1$.

left panel: $\alpha=0$ and $\beta=0$ (bold continues line), $P=0.03558$ (continues line), $\beta=0.05$ (bold dotted line), $P=0.03596$ (dotted line), $\beta=0.15$ (bold dashed line), $P=0.03690$ (dashed line).

middle panel: $\alpha=10^{-4}$ and $\beta=0$ (bold continues line), $P=0.03557$ (continues line), $\beta=0.03$ (bold dotted line), $P=0.03579$ (dotted line), $\beta=0.07$ (bold dashed line), $P=0.03610$ (dashed line).

right panel: $\beta=0.07$ and $\alpha=0.1$ (bold continues line), $P=0.02499$ (continues line), $\alpha=0.5$ (bold dotted line), $P=0.01089$ (dotted line), $\alpha=1$ (bold dashed line), $P=0.00619$ (dashed line).

Next, we have considered the effects of dimensions on the critical behavior. As one can see, the temperature of swallow tail formation, energy gap between two states and minimum of temperature (turning point), critical horizon (middle panels of Figs. 9 and (10), pressure (left panels of Figs. 9] and 10) and $P_{c} r_{c} / T_{c}$ are increasing functions of dimensions. An abnormal behavior for 4-dimension is observed in $G-T$ diagrams for Einstein gravity. This behavior is due to power of $r_{+}$in the last term of Gibbs free energy. The same abnormality could be obtained for the case of GB gravity in 5-dimension which is due to structure of Gibbs free energy, temperature and pressure. In other words, there are terms in these equations that vanish in case of $n=4$.

Finally, we have plotted the coexistence line in which along this curve, small and large black holes have alike temperature and pressure (Figs. 11, 12, and 13). Critical points are located at the end of the coexistence line where above these points the phase transition does not occur. In addition, Fig. 11 indicates that nonlinearity parameter does not significantly affect the coexistence line. For both Einstein and GB gravities as dimension increases the critical pressure and critical temperature increase too (Fig. 12). In case of GB parameter, the critical pressure and critical temperature are decreasing functions of GB parameter (Fig. 13 and table 5).

\section{PHASE TRANSITION POINTS THROUGH HEAT CAPACITY}

Since we have observed an abnormal behavior in plotted phase diagrams, it will be worthwhile to test the existence of reentrant phase transitions through another method. In Ref. [45] a new method for studying critical behaviors was introduced. The method is based on obtaining a relation for thermodynamical pressure by using the denominator of the heat capacity. In other words, by replacing the cosmological constant with its corresponding thermodynamical pressure and solving the denominator of the heat capacity with respect to this pressure a relation is obtained. This relation is different from the pressure which is obtainable from Eq. (15). The maximums of this relation are representing places in which phase transitions take place. Therefore, the maximums in plotting a $P-r_{+}$diagram for this relation will give us critical pressure and horizon radius. This method was employed to obtain critical pressure in several papers which has proven to be an successful one [45, 46].

The heat capacity is obtained by

$$
C_{Q}=\left(\frac{\partial M}{\partial S}\right)_{Q}\left(\frac{\partial^{2} M}{\partial S^{2}}\right)_{Q}^{-1}
$$

Using Eqs. (15) and (16) and replacing cosmological constant with its corresponding relation with pressure and solving its denominator with respect to pressure will lead to a relation for pressure which for economical reasons we will not bring it. We will present the results of this relation by considering mentioned values in different tables in following diagrams (Fig. 14). 
First of all, in absence of the nonlinearity, the plotted diagrams shows existence of only one maximum. The pressure and horizon radius of this maximum is exactly located where phase transition takes place. Interestingly, by adding the nonlinearity to the system, two extrema are observed: a minimum and a maximum. The place of this maximum is exactly where phase transition occurs for mentioned vales for different parameters. But as one can see, no other maximum exists which indicates that, at least no other second order phase transition is observed. In other words, although for specific critical pressure in these diagrams two critical horizons are observed, the type of these critical points are not the same. Therefore, one can conclude that no reentrant phase transitions happens which is consistent with what was observed in coexistence diagrams.

\section{CONCLUSIONS}

In this paper we have considered a quadratic Maxwell invariant as a correction term to the Maxwell Lagrangian. We studied the thermodynamic behavior of these solutions in Einstein and GB gravities. We considered cosmological constant as thermodynamic pressure and related conjugated quantity as volume of the black hole. By doing so, the interpretation of the mass as internal energy was changed into Enthalpy of the system. Therefore, not only the interpretation of the mass of the black hole was changed, but also we extended phase space.

It is worthwhile to make some discussion regarding mass of the black hole in this case. Usually, the interpretation of mass is internal energy. In this point of view mass is a conserved quantity which is representing only the total internal energy. But in case of the new interpretation, mass of the black hole is a combination of internal energy and pressure. In other words, not only the mass of the black holes determines the internal energy and the shape of the black hole, but also we are expecting it to have information regarding interaction of constituents of black holes which is known as pressure of the system. Although, one may state that due to natural properties of black holes, it is impossible to study the interaction of constituents of black holes, one must take the approach of string theory to the matter into consideration. In other words, in context of string theory some attempts were made to study microstates of the black holes and their interpretation which can be extended by considering this point of view.

The volume of the black hole is determined by topological structure of metric. Therefore, one expects that the calculated value of this quantity and the topological structure of the metric be in agreement. This result was obtained in calculation of volume.

In linear electromagnetic field, the critical behavior of the system for both gravities were usual ones. One critical horizon radius was found, hence one phase transition was expected. The formation of the characteristic swallow tail was observed in $G-T$ diagrams. In $T-r_{+}$and $P-r_{+}$diagrams the properties of phase transition were seen. But amazingly, in consideration of additional term the behavior of the system differed completely.

By considering this nonlinear electromagnetic field, the structure of the phase diagrams and the thermodynamic behavior of the system were highly modified. Calculations regarding critical horizon radius lead to existence of two critical horizon radii. It is not unusual to find two critical horizon radii for black holes [11], but in our case the presence of the second critical horizon radius was observed in plotted graphs. In other words, in case of this nonlinear electromagnetic field, contrary to other cases of nonlinear theories, a phase transition and a turning point were observed. The existence of these two points is related to existence of number of critical horizon radius. This result is evident by comparing Maxwell theory with this nonlinear theory. In case of Maxwell theory only one positive critical horizon radius was found which resulted into existence of one phase transition whereas for this case of nonlinear electromagnetic field, two critical horizon radii were found which resulted into existence of the phase transition and turning point.

In case of $G-T$, three points were observed in which the behavior of the system changed in them. These points are representing different phases. In $P-r_{+}$, for $T=T_{c}$, three horizon radii were found with same pressure (critical pressure) which indicates the existence of three phases and one turning point. For $T-r_{+}$, in case of $P=P_{c}$, two horizon radii were found with same temperature (critical temperature). One of these horizons was located on subcritical isobar which is the usual phase transition point and the other one was out of the subcritical isobar.

Another interesting issue was the effects of nonlinearity and GB parameters were opposite of each other. In case of nonlinearity parameter, the smaller critical horizon radius was an increasing function of nonlinearity parameter whereas the larger critical horizon radius was a decreasing function of $\beta$. In this case the smaller critical horizon radius was highly function of nonlinearity parameter comparing to larger critical horizon radius. Therefore, in case of increasing nonlinearity parameter, the distance between these two critical horizon radii decreased. The critical temperature and pressure were increasing functions of nonlinearity parameter whereas $P_{c} r_{c} / T_{c}$ was a decreasing function of it. Interestingly, in case of GB parameter, the smaller critical horizon radius was a decreasing function of GB parameter and the larger critical horizon radius was an increasing function of it. The value of larger critical horizon radius was highly function of variation of GB parameter. Therefore, the distance between two critical horizon radii was an increasing function of GB parameter. As for the critical temperature and pressure, they were decreasing 
functions of GB parameter. In this case, $P_{c} r_{c} / T_{c}$ was also a decreasing function of GB parameter.

These two parameters $(\alpha$ and $\beta)$ are describing two aspects of the black holes; gravitational and matter fields. In case of increasing $\beta$, the nonlinearity behavior of the system increases. In context of electromagnetic tensor, increasing this parameter leads to decreasing value of this tensor (8). Therefore, the values of the electromagnetic tensor are decreasing functions of nonlinearity parameter. This fact leads to a conclusion that increasing the power of nonlinearity leads to decreasing power of electromagnetic field. But in case of GB parameter, by increasing its value, the power of the gravitational field increases. In case of this generalization due to considering higher orders of curvature scalar, we are increasing the gravitational force. As one can see, increasing gravitational force causes the critical temperature and pressure decrease which indicating that in this case phase transition is taking place in lower temperature, hence lower energy. Therefore, increasing the power of the gravitational force causes the system to have higher value of internal energy. This can also be seen in studying entropy of the GB gravity. As one can see, in case of spherical symmetric, entropy is an increasing function of GB parameter. In comparing GB and Einstein gravities, it is evident that GB gravity has higher entropy. Having higher entropy means that system has higher internal energy which causes the system to have phase transition in lower temperature. This result was found in studying phase diagrams of the GB and Einstein diagrams.

By what was mentioned in last paragraph one can conclude following results: first of all generalization of GB gravity causes the internal energy of the system increases and the black hole has phase transition faster comparing to Einstein gravity. Therefore, in this theory black holes need to absorb less mass to have phase transition. Second, the entropy may be a function of the complexity of the black holes structure. Considering higher orders of curvature scalar causes the system to have more complicated structure. Therefore, it is arguable that the entropy and the complexity of the structure of the system are related to each other. In other words, complexity of the structure is a measurement for entropy of the system. Nonlinearity of the system and gravitational force are two opposing factors. They are decreasing each others effects and at some points they may cancel each others effects. This argument is stating that it may be possible to fix parameters in a way which system has critical values same as ones in Einstein-Maxwell theory.

Due to existence of the abnormal behavior in phase diagrams a question regarding the existence of reentrant phase transitions could rise which was answered through two methods. The coexistence diagrams and a new method showed that only one of the critical horizons are representing second order phase transition, while the other one could be another type of the phase transition.

As for the effects of the dimensions, the smaller critical horizon, pressure, temperature and $P_{c} r_{c} / T_{c}$ were increasing functions of dimensions whereas, the subcritical isobars and larger critical horizon radius were decreasing functions of it. This behavior is indicating that the higher dimensional black holes need to absorb more mass to have phase transition. It is worthwhile to mention that case $n=3$ for Einstein gravity and $n=4$ for GB gravity are considered to be special cases in these gravities. As for the GB gravity, in this specific dimension, some terms in Eqs. (15) and (25) vanish. In case of Einstein gravity, it is evident from Eq. (25) that for case of $n=3$ the power of the horizon radius is different from other dimensions in a way which causes the Gibbs free energy to have abnormal behavior which was observed in studying phase diagrams.

As it was pointed out, considering whether we are working in context of stringy correction or classical Born-Infeld theories, the results may be different. As it was seen, in case of later correction, there was a limiting point which for specific values makes the system never acquire non-Van der Waals behavior, whereas in case of stringy corrected electromagnetic field such limitation did not exist and the system would have non-Van der Waals like behavior. This points out that in stringy corrected version of the theory, different terms have different dominant regions which correspondingly modify phase structure and phase transitions of the solutions.

Finally, it is worthwhile to mention that regarding string theory, the quadratic Maxwell invariant arises independent of Born-Infeld electromagnetic field. If one conducts the study that was done in this paper by motivations of string theory (not correction to Maxwell theory), there will be no limitation for considered values. Therefore, one can omit vertical limiting lines in plotted graphs. In addition, we should note that modification in phase diagrams, previously was obtained by changing the gravitational part of action. Here in this paper, the modification of matter field caused the abnormal behavior of the phase diagrams. 


\section{Acknowledgments}

We also thank the Shiraz University Research Council. This work has been supported financially by the Research

Institute for Astronomy and Astrophysics of Maragha, Iran.

[1] S. W. Hawking, Phys. Rev. Lett. 26 (1971) 1344;

J. D. Bekenstein, Phys. Rev. D 7 (1973) 2333;

J. M. Bardeen, B. Carter and S. W. Hawking, Commun. Math. Phys. 31 (1973) 161.

[2] P. Hut, Mon. Not. Roy. Astron. Soc. 180 (1977) 379;

P. C. W. Davies, Rep. Prog. Phys. 41 (1978) 8.

[3] M. W. Zemansky and R. H. Dittman, Heat and thermodynamics: an intermediate textbook, McGraw-Hill, U.S.A. (1997).

[4] R. Banerjee, S. Ghosh and D. Roychowdhury, Phys. Lett. B 696 (2011) 156.

[5] G. Ruppeiner, Phys. Rev. A 20 (1979) 1608.

[6] M. Cvetic and S. S. Gubser, JHEP 04 (1999) 024;

M. M. Caldarelli, G. Cognola and D. Klemm, Class. Quantum Gravit. 17 (2000) 399.

[7] C. V. Johnson, Class. Quantum Gravit. 31 (2014) 205002;

B. P. Dolan, Mod. Phys. Lett. A, 30, (2015) 1540002;

B. P. Dolan, JHEP 10 (2014) 179.

[8] D. Kastor, S. Ray and J. Traschen, Class. Quantum Gravit. 26 (2009) 195011;

M. Urano, A. Tomimatsu and H. Saida, Class. Quantum Gravit. 26 (2009) 105010;

B. Dolan, Class. Quantum Gravit. 28 (2011) 125020;

B. P. Dolan, Class. Quantum Gravit. 28 (2011) 235017;

B. P. Dolan, Phys. Rev. D 84 (2011) 127503.

[9] A. Chamblin, R. Emparan, C. Johnson and R. Myers, Phys. Rev. D 60 (1999) 064018;

A. Chamblin, R. Emparan, C. Johnson and R. Myers, Phys. Rev. D 60 (1999) 104026.

[10] N. Goldenfeld, Lectures on Phase Transitions and the Renormalization Group. Westview Press, New York, (1992).

[11] C. Peca and J. Lemos, Phys. Rev. D 59 (1999) 124007;

G. Gibbons, M. Perry and C. Pope, Class. Quantum Gravit. 22 (2005) 1503;

S. Wang, S. Q. Wu, F. Xie and L. Dan, Chin. Phys. Lett. 23 (2006) 1096;

Y. Sekiwa, Phys. Rev. D 73 (2006) 084009;

G. L. Cardoso and V. Grass, Nucl. Phys. B 803 (2008) 209;

H. Elvang, R. Emparan and P. Figueras, JHEP 05 (2007) 056;

R. Emparan, T. Harmark, V. Niarchos, N. A. Obers and M. Rodriguez, JHEP 10 (2007) 110;

O. Dias, P. Figueras, R. Monteiro, J. E. Santos and R. Emparan, Phys. Rev. D 80 (2009) 111701;

R. Banerjee, S. K. Modak and S. Samanta, Phys. Rev. D 84 (2011) 064024;

C. Niu, Y. Tian and X. N. Wu, Phys. Rev. D 85 (2012) 024017;

Y. D. Tsai, X. Wu and Y. Yang, Phys. Rev. D 85 (2012) 044005;

M. S. Ma, H. H. Zhao, L. C. Zhang and R. Zhao, Int. J. Mod. Phys. A 29 (2014) 1450050;

S. Chen, X. Liu, C. Liu and J. Jing, Chin. Phys. Lett. 30 (2013) 060401;

R. Zhao, H. H. Zhao, M. S. Ma and L. C. Zhang, Eur. Phys. J. C 73 (2013) 2645;

J. X. Mo, X. X. Zeng, G. Q. Li, X. Jiang and W. B. Liu, JHEP 10 (2013) 056;

M. B. J. Poshteh, B. Mirza and Z. Sherkatghanad, Phys. Rev. D 88 (2013) 024005;

S. A. Hosseini Mansoori and B. Mirza, Eur. Phys. J. C 74 (2014) 2681;

B. Mirza and Z. Sherkatghanad, Phys. Rev. D 90 (2014) 084006.

[12] S. Hendi and M. Vahidinia, Phys. Rev. D 88 (2013) 084045.

[13] S. W. Hawking and D. N. Page, Commun. Math. Phys. 87 (1983) 577.

[14] D. Kubiznak and R. B. Mann, JHEP 07 (2012) 033;

S. Gunasekaran, D. Kubiznak and R. Mann, JHEP 11 (2012) 110.

[15] S. W. Wei and Y. X. Liu, Phys. Rev. D 90 (2014) 044057;

S. W. Wei and Y. X. Liu, Phys. Rev. D 87 (2013) 044014;

D. Zou, Y. Liu and B. Wang, Phys. Rev. D 87 (2013) 044014;

W. Xua, H. Xub and L. Zhaoc, Eur. Phys. J. C 74 (2014) 2970;

S. H. Hendi, S. Panahiyan and B. Eslam Panah, Prog. Theor. Exp. Phys. 2015, $103 E 01$ (2015);

[16] R. G. Cai, L. M. Cao, L. Li and R. Q. Yang, JHEP 09 (2013) 005.

[17] A. M. Frassino, D. Kubiznak, R. B. Mann and F. Simovic, JHEP 09 (2014) 080;

R. A. Hennigar, W. G. Brenna and R. B. Mann, JHEP 07 (2015) 077.

[18] M. Born and L. Infeld, Proc. Roy. Soc. Lond. A 143 (1934) 410;

M. Born and L. Infeld, Proc. Roy. Soc. Lond. A 144 (1934) 425.

[19] B. Hoffmann, Phys. Rev. 47 (1935) 877.

[20] W. Yao and J. Jing, JHEP 05 (2014) 058; 
S. Gangopadhyay, Mod. Phys. Lett. A 29 (2014) 1450088;

S. Gangopadhyay and D. Roychowdhury, JHEP 05 (2012) 156;

S. Gangopadhyay and D. Roychowdhury, JHEP 05 (2012) 002;

J. Jing, L. Wang, Q. Pan and S. Chen, Phys. Rev. D 83 (2011) 066010;

J. Jing and S. Chen, Phys. Lett. B 686 (2010) 68.

[21] H. Q. Lu, L. M. Shen, P. Ji, G. F. Ji and N. J. Sun, Int. J. Theor. Phys. 42 (2003) 837;

M. H. Dehghani and S. H. Hendi, Gen. Relativ. Gravit. 41 (2009) 1853;

E. F. Eiroa and G. F. Aguirre, Eur. Phys. J. C 72 (2012) 2240.

[22] S. H. Hendi, Adv. High Energy Phys. 2014 (2014) 697863.

[23] M. H. Dehghani, N. Alinejadi and S. H. Hendi, Phys. Rev. D 77 (2008) 104025;

M. H. Dehghani and S. H. Hendi, Phys. Rev. D 73 (2006) 084021;

M. Allahverdizadeh, S. H. Hendi, J. P. S. Lemos and A. Sheykhi, Int. J. Mod. Phys. D 23 (2014) 1450032;

R. Banerjee and D. Roychowdhury, Phys. Rev. D 85 (2012) 104043;

A. Lala and D. Roychowdhury, Phys. Rev. D 86 (2012) 084027;

R. Banerjee and D. Roychowdhury, Phys. Rev. D 85 (2012) 044040;

P. Li, R. H. Yue and D. C. Zou, Commun. Theor. Phys. 56 (2011) 845;

D. C. Zou, Z. Y. Yang, R. H. Yue and P. Li, Mod. Phys. Lett. A 26 (2011) 515;

A. Ghodsi and D. M. Yekta, Phys. Rev. D 83 (2011) 104004;

R. G. Cai and Y. W. Sun, JHEP 09 (2008) 115;

S. H. Mazharimousavi, M. Halilsoy and Z. Amirabi, Phys. Rev. D 78 (2008) 064050;

W. A. Chemissany, M. de Roo and S. Panda, Class. Quantum Gravit. 25 (2008) 225009;

Y. S. Myung, Y. W. Kim and Y. J. Park, Phys. Rev. D 78 (2008) 084002;

Y. S. Myung, Y. W. Kim and Y. J. Park, Phys. Rev. D 78 (2008) 044020;

O. Miskovic and R. Olea, Phys. Rev. D 77 (2008) 124048;

I. Zh. Stefanov, S. S. Yazadjiev and M. D. Todorov, Phys. Rev. D 75 (2007) 084036;

S. Fernando, Phys. Rev. D 74 (2006) 104032;

R. G. Cai, D. W. Pang and A. Wang, Phys. Rev. D 70 (2004) 124034;

M. Aiello, R. Ferraro and G. Giribet, Phys. Rev. D 70 (2004) 104014;

T. K. Dey, Phys. Lett. B 595 (2004) 484;

T. Tamaki, JCAP 05 (2004) 004;

S. Fernando and D. Krug, Gen. Relativ. Gravit. 35 (2003) 129;

M. Wirschins, A. Sood and J. Kunz, Phys. Rev. D 63 (2001) 084002;

M. Cataldo and A. Garcia, Phys. Lett. B 456 (1999) 28.

[24] S. H. Hendi, J. Math. Phys. 49 (2008) 082501;

M. H. Dehghani, S. H. Hendi, A. Sheykhi and H. Rastegar Sedehi, JCAP 02 (2007) 020;

M. H. Dehghani and S. H. Hendi, Int. J. Mod. Phys. D 16 (2007) 1829;

M. H. Dehghani and H. Rastegar Sedehi, Phys. Rev. D 74 (2006) 124018;

S. H. Hendi, Phys. Rev. D 82 (2010) 064040;

D. J. Cirilo-Lombardo, Gen. Relativ. Gravit. 37 (2005) 847;

V. Ferrari, L. Gualtieri, J. A. Pons and A. Stavridis, Mon. Not. Roy. Astron. Soc. 350 (2004) 763.

[25] E. Fradkin and A. Tseytlin, Phys. Lett. B 163 (1985) 123;

R. Matsaev, M. Rahmanov and A. Tseytlin, Phys. Lett. B 193 (1987) 205;

C. Callan, C. Lovelace, C. Nappi and S. Yost, Nucl. Phys. B 308 (1988) 221;

O. Andreev and A. Tseytlin, Nucl. Phys. B 311 (1988) 221;

R. Leigh, Mod. Phys. Lett. A 04 (1989) 2767.

[26] H. H. Soleng, Phys. Rev. D 52 (1995) 6178.

[27] S. H. Hendi, JHEP 03 (2012) 065.

[28] S. H. Hendi, Ann. Phys. (N.Y.) 333 (2013) 282;

S. H. Hendi and A. Sheykhi, Phys. Rev. D 88 (2013) 044044;

S. H. Hendi, Ann. Phys. (N.Y.) 346 (2014) 42;

S. H. Hendi, Adv. High Energy Phys. 2014 (2014) 697914.

[29] D. H. Delphenich, Nonlinear electrodynamics and QED, [arXiv: hep-th/0309108;

D. H. Delphenich, Nonlinear optical analogies in quantum electrodynamics, [arXiv: hep-th/0610088];

W. Heisenberg and H. Euler, Z. Phys. 98 (1936) 714. Translation by: W. Korolevski and H. Kleinert, Consequences of Dirac's Theory of the Positron, physics/0605038;

J. Schwinger, Phys. Rev. 82 (1951) 664;

P. Stehle and P. G. DeBaryshe, Phys. Rev. 152 (1966) 1135.

[30] V. A. De Lorenci and M. A. Souza, Phys. Lett. B 512 (2001) 417;

V. A. De Lorenci and R. Klippert, Phys. Rev. D 65 (2002) 064027;

M. Novello and E. Bittencourt, Phys. Rev. D 86 (2012) 124024;

M. Novello et al., Class. Quantum Gravit. 20 (2003) 859.

[31] E. Ayon-Beato and A. Garcia, Gen. Relativ. Gravit. 31 (1999) 629.

[32] E. Ayon-Beato and A. Garcia, Phys. Lett. B 464 (1999) 25;

H. P. Oliveira, Class. Quantum Gravit. 11 (1994) 1469; 
D. Palatnik, Phys. Lett. B 432 (1998) 287;

E. Ayon-Beato and A. Garcia, Phys. Rev. Lett. 80 (1998) 5056.

[33] V. A. De Lorenci, R. Klippert, M. Novello and J. M. Salim, Phys. Rev. D 65 (2002) 063501;

I. Dymnikova, Class. Quantum Gravit. 21 (2004) 4417;

C. Corda and H. J. Mosquera Cuesta, Mod. Phys. Lett A 25 (2010) 2423;

C. Corda and H. J. Mosquera Cuesta, Astropart. Phys. 34 (2011) 587.

[34] H. J. Mosquera Cuesta and J. M. Salim, Mon. Not. Roy. Astron. Soc. 354 (2004) L55;

H. J. Mosquera Cuesta and J. M. Salim, Astrophys. J. 608 (2004) 925;

Z. Bialynicka-Birula and I. Bialynicka-Birula, Phys. Rev. D 2 (1970) 2341.

[35] R. Gopakumar, S. Minwalla, N. Seiberg, A. Strominger, JHEP 08 (2000) 008;

T. Tamaki and K. Maida, Phys. Rev. D 62 (2000) 084041;

S. Kar and S. Majumdar, Int. J. Mod. Phys. A 21 (2006) 6087.

[36] M. Brigante, H. Liu, R. C. Myers, S. Shenker and S. Yaida, Phys. Rev. D 77 (2008) 126006;

Y. Kats and P. Petrov, JHEP 01 (2009) 044;

P. Kovtun, D. T. Son and A. O. Starinets, JHEP 10 (2003) 064;

X. H. Ge, Y. Matsuo, F. W. Shu, S. J. Sin and T. Tsukioka, JHEP 10 (2008) 009.

[37] A. Tseytlin, Nucl. Phys. B 276 (1985) 391;

D. J. Gross and J. H. Sloan, Nucl. Phys. B 291 (1987) 41;

Y. Kats, L. Motl and M. Padi, JHEP 12 (2007) 068;

D. Anninos and G. Pastras, JHEP 07 (2009) 030;

R. G. Cai, Z. Y. Nie and Y. W. Sun, Phys. Rev. D 78 (2008) 126007;

N. Seiberg and E. Witten, JHEP 09 (1999) 032;

E. Bergshoeff, E. Sezgin, C. Pope and P. Townsend, Phys. Lett. B 188 (1987) 70.

[38] S. H. Hendi and M. Momennia, Eur. Phys. J. C 75 (2015) 54.

[39] S. H. Hendi, B. Eslam Panah, M. Momennia and S. Panahiyan, Eur. Phys. J. C 75 (2015) 457.

[40] S. H. Hendi, Eur. Phys. J. C 73 (2013) 2634;

S. H. Hendi, S. Panahiyan and B. Eslam Panah, Eur. Phys. J. C 75 (2015) 296.

[41] S. H. Hendi and S. Panahiyan, Phys. Rev. D 90 (2014) 124008.

[42] S. H. Hendi Int. J. Mod. Phys. D 24 (2015) 1550040.

[43] Y. C. Bruhat, J. Math. Phys. 29 (1988) 1891;

X. O. Camanho and J. D. Edelstein, JHEP 04 (2010) 007;

X. O. Camanho and J. D. Edelstein, JHEP 06 (2010) 099;

K. Izumi, Phys. Rev. D 90 (2014) 044037;

X. O. Camanho, J. D. Edelstein, J. Maldacena and A. Zhiboedov, arXiv:1407.5597;

G. D'Appollonio, P. Di Vecchia, R. Russo and G. Veneziano, JHEP 05 (2015) 144.

[44] M. A. Ainou, Eur. Phys. J. C 75, (2015) 34;

M. A. Ainou, Phys. Rev. D 91 (2015) 064049.

[45] S. H. Hendi, S. Panahiyan and B. Eslam Panah, Int. J. Mod. Phys. D 25 (2016) 1650010.

[46] S. H. Hendi, B. Eslam Panah and S. Panahiyan, JHEP 11 (2015) 157;

S. H. Hendi, S. Panahiyan and B. Eslam Panah, JHEP 01 (2016) 129.

[47] R. Aldrovandi, J. P. Beltran Almeida and J. G. Pereira, Int. J. Mod. Phys. D 13 (2004) 2241.

[48] D. Kastor, S. Ray, and J. Traschen, Class. Quantum Gravit. 27 (2010) 235014;

D. C. Zou, S. J. Zhang and B. Wang, Phys. Rev. D 89 (2014) 044002;

Z. Sherkatghanad, B. Mirza, Z. Mirzaeyan and S. A. H. Mansoori, arXiv:1412.5028.

[49] B. P. Dolan, A. Kostouki, D. Kubiznak and R. B. Mann, arXiv:1407.4783. 